1 Title: Domain Binding and Isotype Dictate the Activity of Anti-human OX40

2 Antibodies

3

4 Authors: Jordana Griffiths ${ }^{1}$, Khiyam Hussain ${ }^{1}$, Hannah L Smith ${ }^{1}$, Theodore Sanders ${ }^{1}$ Kerry

5 Cox $^{1}$, Monika Semmrich², Linda Martensson², Jinny Kim², Tatyana Inzhelevskaya ${ }^{1}$, Chris

6 Penfold $^{1}$, Alison L Tutt ${ }^{1}$, Ian M Mockridge ${ }^{1}$, Claude HT Chan ${ }^{1}$, Vikki English ${ }^{1}$, Ruth R

7 French $^{1}$, Ingrid Teige ${ }^{2}$, Aymen Al-Shamkhani ${ }^{1}$, Martin J Glennie ${ }^{1}$, Bjorn Frendeus ${ }^{2}$, Jane E

8 Willoughby $^{1 \dagger}$, Mark S Cragg ${ }^{1{ }^{+\star}}$

9

$10{ }^{1}$ Antibody and Vaccine Group, Centre for Cancer Immunology, Cancer Sciences Unit,

11 Faculty of Medicine, University of Southampton, Tremona Road, Southampton SO16 6YD,

12 UK. ${ }^{2}$ Preclinical Research, Biolnvent International AB, Sölvegatan 41, 22370 Lund, Sweden.

13

14 These authors contributed equally to this work

15

16

${ }^{*}$ Corresponding author: Professor Mark Cragg, Antibody and Vaccine Group, MP127, Centre for Cancer Immunology, Cancer Sciences, Faculty of Medicine, University Hospital

Southampton, Tremona Road, Southampton, SO16 6YD, UK. msc@soton.ac.uk.

Running Title: Isotype and epitope dictate OX40 mAb activity

Keywords: OX40, isotype, receptor agonism, therapeutic activity

Word Count: 6452 
36 Abbreviations:

37 ACK

Ammonium Chloride Potassium

38

ADCC

Antibody dependent cellular cytotoxicity

39

ADCP

Antibody dependent cellular phagocytosis

40 CDC

Complement dependent cytotoxicity

41 CRD

Cysteine rich domains (CRDs)

42 ECD

Extracellular domain

43 Eomes

Eomesodermin

44 I.P.

Intraperitoneal

45 I.V.

Intravenous

$46 \mathrm{KI}$

Knock-in

$47 \mathrm{KO}$

Knock-out

$48 \quad \mathrm{mAb}$

Monoclonal antibody

49 MPECS

Memory precursor cells

50 NOG

NOD/Shi-scid/IL-2R $\gamma^{\text {null }}$

$51 \quad$ NSG

NOD scid gamma

52 PBMCs

Peripheral blood mononuclear cells

53 PCR

Polymerase chain reaction

54 SCID

Severe combined immune deficient

55 SLECS

Short lived effector cells

56 SPR

Surface Plasmon Resonance

57 tdLN

Tumour draining lymph node

58 TILS

Tumour inflitrating lymphocytes

59 TME

Tumour microenvironment

60 TNFR

Tumour necrosis factor receptors

61 WT

Wildtype

62

63

64

65

66

67

68

69 


\section{Title: Domain Binding and Isotype Dictate the Activity of Anti-human OX40 Antibodies}

Authors: Jordana Griffiths ${ }^{1}$, Khiyam Hussain ${ }^{1}$, Hannah L Smith ${ }^{1}$, Theodore Sanders ${ }^{1}$ Kerry Cox ${ }^{1}$, Monika Semmrich ${ }^{2}$, Linda Martensson ${ }^{2}$, Jinny Kim $^{1}$, Tatyana Inzhelevskaya ${ }^{1}$, Chris Penfold ${ }^{1}$, Alison L Tutt' ${ }^{1}$, lan M Mockridge ${ }^{1}$, Claude HT Chan ${ }^{1}$, Vikki English ${ }^{1}$, Ruth R French ${ }^{1}$, Ingrid Teige ${ }^{2}$, Aymen AI-Shamkhani ${ }^{1}$, Martin J Glennie ${ }^{1}$, Bjorn Frendeus ${ }^{2}$, Jane E Willoughby ${ }^{1 \dagger}$, Mark S Cragg ${ }^{1 \dagger^{\star}}$

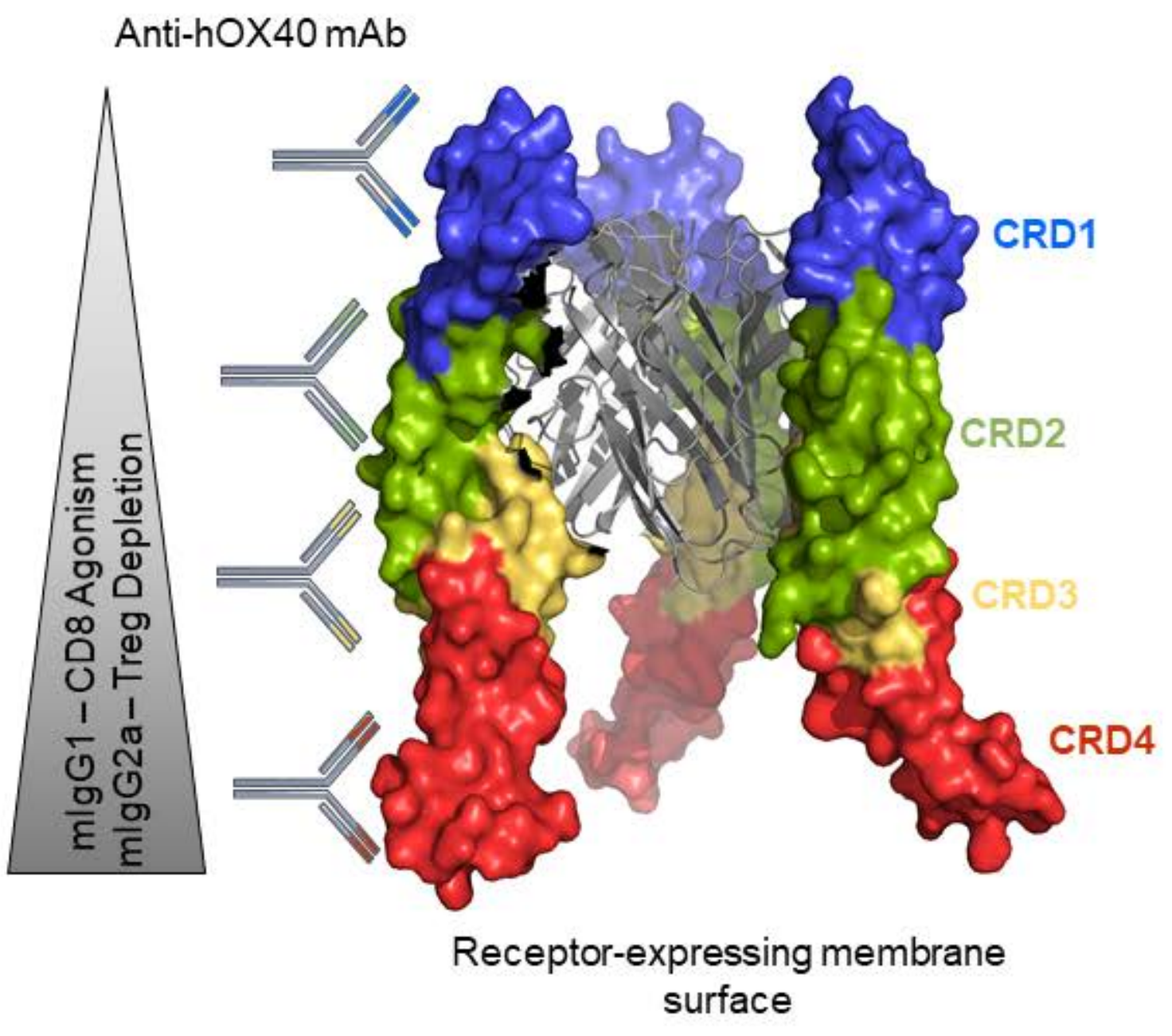

Isotype and epitope dictate OX40 mAb activity;

- $\mathrm{mlgG1}$ promotes direct agonism on CD8+T cells

- mlgG2a depletes hOX40 expressing Tregs

- Strength of both agonism and depleting activity is greatest with membrane proximal binding $\mathrm{mAb}$

- Provides insight into how anti-hOX 40 mAb could be improved for clinical efficacy 


\section{Abstract}

Background: Previous data suggests that anti-OX40 mAb can elicit anti-tumour effects in mice through deletion of Tregs. However, OX40 also has powerful costimulatory effects on T cells which could evoke therapeutic responses. Human trials with anti-OX40 antibodies have shown that these entities are well tolerated but to date have delivered disappointing clinical responses, indicating that the rules for the optimal use of anti-human OX40 (hOX40) antibodies is not yet fully understood. Changes to timing and dosages may lead to improved outcomes, however here we focus on addressing the role of agonism vs depleting activity in determining therapeutic outcomes. We investigated a novel panel of anti-hOX40 mAb to understand how these reagents and mechanisms may be optimised for therapeutic benefit.

Methods: This study examines the binding activity and in vitro activity of a panel of anti-hOX40 antibodies. They were further evaluated in several in vivo models to address how isotype and epitope determine mechanism of action and efficacy of anti-hOX40 mAb.

Results: Binding analysis revealed the antibodies to be high affinity with epitopes spanning all four cysteine rich domains of the OX40 extracellular domain. In vivo analysis showed that their activities relate directly to two key properties: 1 ) isotype with mlgG1 mAb evoking receptor agonism and CD8+ T cell expansion and mlgG2a mAb evoking deletion of Treg and; 2) epitope - with membrane-proximal mAb delivering more powerful agonism. Intriguingly, both isotypes acted therapeutically in tumour models by engaging these different mechanisms.

Conclusions: These findings highlight the significant impact of isotype and epitope on the modulation of anti-hOX40 mAb therapy, and indicate that CD8+ T cell expansion or Treg depletion might be preferred according to the composition of different tumours. As many of the current clinical trials using OX40 antibodies are now using combination therapies, this understanding of how to manipulate therapeutic activity will be vital in directing new combinations that are more likely to improve efficacy and clinical outcomes. 


\section{Introduction}

The use of immunomodulating monoclonal antibodies (mAb) to generate anti-tumour immune responses offers an exciting approach to cancer immunotherapy. mAb against immune checkpoint inhibitors such as Ipilimumab and Nivolumab, which target the co-inhibitory receptors CTLA-4 and PD-1, respectively, pioneered this approach and have demonstrated success in treating a number of previously untreatable cancers $[1,2]$. However, many patients do not respond to these reagents and additional therapeutic strategies are required. Agonistic mAb targeting costimulatory receptors have emerged as targets for clinical development, in particular tumour necrosis factor receptors (TNFR) superfamily members such as CD40 [3], 41BB [4], and OX40 [5-7]. However Freeman et al identified a intratumoural Treg signature which included TNFR family members with the hypothesis that they could be targeted instead by depleting antibodies in order to generate therapy [8]. TNFR family members are typically characterised by an extracellular domain (ECD) consisting of several cysteine rich domains (CRDs) which allow for binding of their respective trimeric ligands leading to receptor clustering and downstream signalling [9]. mAb targeting such receptors have been shown to depend on their interaction with the inhibitory Fc $\gamma \mathrm{R}(\mathrm{Fc} \gamma \mathrm{RIIB})$ to generate sufficient cross-linking and resultant agonistic activity $[10,11]$. More recently, the ability of a number of TNFR mAb to cause deletion of Tregs via engagement of activatory Fc $\gamma \mathrm{R}$ has been demonstrated $[12,13]$. The anti-mouse OX40 mAb, OX86, has previously been shown to enhance effector T cell proliferation and survival leading to successful therapeutic outcomes in pre-clinical models [5, 14]. Recently it has also been demonstrated to be capable of deleting Tregs in an activatory Fc $\gamma R$-dependent manner [12]. This effect was directly influenced by isotype, with mlgG2a showing greater depleting capacity than the native rlgG1 isotype. Interestingly Tregs were preferentially deleted over effector $T$ cells which correlated with mOX40 expression on these cells [12].

Work on several TNFRs has further highlighted the importance of the region targeted by the antibody in influencing the type and strength of effector function [15-17]. For anti-CD40 mAb, the membrane distal CRD1-binding mAb were shown to be strong agonists of CD40 with membrane proximal mAb less potent [16]. Furthermore, mAb binding CRD2-4 blocked CD4OL and were potent antagonists. Additionally anti-4- 
137

1BB mAb which bound membrane proximal domains engaged in more effective CDC and ADCC killing mechanisms with ADCP less affected [15] . Moreover, Zhang et al. reported that mAb binding to mouse ( $\mathrm{m}) \mathrm{OX} 40$ which blocked ligand binding and bound CRD2, or bound at the membrane proximal domain (CRD4), provide stronger agonistic and anti-tumour activity than mAb binding CRD1 and 3 [17]. These results differed from those seen for $\mathrm{hCD} 40$, highlighting that the functional effects of mAb domain binding are likely to require assessment for each of the TNFR family members and validation for each species.

Given these discrepancies, we explored the optimal domain binding and isotype for a novel panel of anti-human (h)OX40 mAb which collectively bound to all 4 CRDs of the ECD of hOX40. We evaluated their function in vitro and in vivo as both mlgG1 and mlgG2a isotypes. Using a novel hOX40 knock-in (KI) mouse we found that mlgG1 mAb were agonistic and engendered memory responses, whereas mlgG2a $\mathrm{mAb}$ had depleting activity with poorer memory recall responses. The strength of these effector functions appeared to correlate with domain binding; those mAb which bound to the most membrane proximal domain (CRD4) which did not block ligand binding, showed the strongest agonistic activity as mlgG1 as well as the most potent depleting activity as mlgG2a. This data highlights how mAb to different TNFR (and different species) exhibit different requirements in relation to optimal domain binding and effector function and indicate how more active anti-hOX40 mAb might be developed.

\section{Results}

\section{hOX40KI mice express hOX40 and develop normally}

To investigate the immunotherapeutic potential of a new panel of anti-hOX40 mAb we generated a new knock-in $(\mathrm{KI})$ mouse, designed to express hOX40 extracellular domain and mOX40 transmembrane and intracellular domains (Supplementary Fig. 1A). PCR confirmed integration of the construct and identified WT, hOX4OKI ${ }^{+/}$and hOX40 $\mathrm{KI}^{+/+}$mice (Supplementary Fig. 1B). Analysis of OX40 surface expression (mouse and human) on resting splenocyte $\mathrm{T}$ cells from $\mathrm{WT}$, hOX $40 \mathrm{KI}^{+/-}$and hOX $40 \mathrm{KI}^{+/+}$mice confirmed that the chimeric receptor was expressed at the cell 
171 surface on relevant cell types (Fig. 1A and B) and in a gene dose-dependent manner

172 (Supplementary Fig. 1C). Expression of OX40 in all three genotypes was largely

173 restricted to $T$ cell lineages (Fig. 1A, B and Supplementary Fig. 1D). In line with

174 previous reports [12, 18-20], a hierarchal expression pattern amongst the $T$ cell

175 subsets was observed in all genotypes with expression highest on Tregs followed by

176 CD4+ effectors, with limited expression on resting CD8+ T cells (Fig. 1A and B). To

177 address whether the hOX40KI ${ }^{+/+}$mice represented a functional model to study the

178 OX40L:OX40 axis, we performed SPR analysis of mOX40L and hOX40L binding to

179 hOX40 (Supplementary Fig. 1E). Both mOX40L and hOX40L bound similarly to

180 hOX40, in agreement with earlier studies showing that mOX40L engages the same

181 domains on hOX40 as hOX40L [21]. Furthermore, OX40 KO mice are reported to

182 have a subtle defect in Treg numbers (reviewed in [22]). However expression of the

183 chimeric receptor did not affect normal immune cell development (Supplementary

184 Fig. 1F), further indicating that OX40L:OX40 signalling axis is intact in these

185 hOX40KI mice.

186

187 Consistent with previous findings [23], activated splenocytes isolated from WT, $188 \mathrm{hOX} 40 \mathrm{KI}^{+/-}$and hOX40KI+/+ mice showed peak OX40 expression between 24-48 189 hours post activation (Fig. 1C). The kinetics of expression of hOX40 on hOX40KI+/190 and hOX40KI ${ }^{+/+}$splenocytes correlated with that of hOX40 on activated human 191 PBMCs (Fig. 1D). A hierarchy of expression was also observed in both activated 192 splenocytes and hPBMCs with greatest expression detectable on Tregs (Fig. 1C and 193 1D). Samples taken from human cancer patients also showed the same pattern 194 (Tregs > CD4+ effectors > CD8+ T cells) with highest OX40 expression on T cells 195 isolated from tumour sites (Fig. 1E). Collectively, these results validated the use of 196 the hOX40KI model to assess anti-hOX40 mAb.

\section{Generation and characterisation of a panel of anti-hOX40 mAb}

199 A panel of seven anti-hOX40 mAb were subsequently generated by conventional 200 hybridoma technology and characterised. All mAb displayed a high affinity for hOX40 201 (KD values between $10^{-9}-10^{-10} \mathrm{M}$ ) as determined by SPR (Supplemental Fig. 2A) and 202 did not bind mOX40 (Supplementary Fig 2B and C). The crystal structure of hOX40 203 indicates four CRDs [21]. To determine which of these the anti-hOX40 mAb bound, 204 hOX40 domain mutants were generated, lacking CRD1, CRD1+2 or CRD1, 2+3 (Fig. 
2A). A hCD20 epitope tag and mOX40 CRD3 domain were added to the final construct to stabilise its expression. Across the panel, at least one antibody bound to each of the different domains (Fig. 2B and Supplemental Fig. 2D). Cross-blocking experiments showed that anti-hOX40 mAb which bound to the same domain blocked the binding of one another, whereas mAb binding to different CRDs could bind simultaneously (Fig. 2C). The domain binding for each antibody is summarised in Fig. 2D. Finally, SPR analysis revealed that only mAb binding to CRD4 were able to bind in the presence of the ligand (Fig. 2E and Supplemental Fig 2E), indicating ligand binding blocks binding of mAb that recognise epitopes in CRD1-3.

Antibodies were then assessed for their ability to augment sub-optimal anti-CD3 mediated proliferation of hPBMC. All of the mlgG1 mAb increased proliferation of CD8+ $T$ cells (Fig. 2F) whereas mlgG2a mAb reduced proliferation. Differences between mlgG1 and mlgG2a isotypes have previously been reported for other TNFR family members $[24,25]$ and so we class-switched the mAb so that both mlgG1 and mlgG2a isotypes were available. A similar trend was seen in hOX40KI splenocytes stimulated with anti-hOX40 mAbs with mlgG1 mAb showing a trend towards an increase in proliferation whilst mlgG2a mAb showed a clear reduction in proliferation (Supplementary Fig 3).

\section{anti-hOX40 mlgG1 mAb are agonistic in vivo}

To investigate the ability of the anti-hOX40 mAb to cause antigen-specific $C D 8^{+} \mathrm{T}$ cell expansion in vivo we used the OT-I model whereby antigen specific T cells are transferred into naive recipients. hOX40K $\mathrm{KI}^{+-}$OT-I T cells, which recognise the OVA $257-264 / \mathrm{H}-2 \mathrm{~K}^{\mathrm{b}}$ complex, were adoptively transferred into hOX40 $\mathrm{KI}^{+/+}$mice before vaccination with OVA and administration of anti-hOX40 mAb as either mlgG1 or mlgG2a (Fig. 3A). Both mlgG1 and mlgG2a anti-hOX40 mAb expanded antigenspecific CD8+ OT-I T cells in blood compared to OVA alone and to a similar extent with the exception of SAP 28-2 which was notably weaker as a mlgG2a (Fig. 3B and Supplementary Fig. 4A). Despite reaching similar frequencies at the peak of the primary response, upon re-challenge with SIINFEKL peptide alone, a significantly smaller recall response was seen in mice that had received mlgG2a antibodies (Fig. 3B and Supplementary Fig. 4B). Given the time between antibody administration and rechallenge with SIINFEKL peptide, it is likely this lack of recall reflects responses 
initiated during the priming stage as opposed to any effects of mAb persisting from the initial challenge. The frequency of OT-I cells pre-recall in mlgG1 treated mice strongly correlated with the strength of the recall peak (Supplementary Fig. 4C), however due to the lack of a recall response seen in the mlgG2a it is not possible to determine if this is also the case for the mlgG2a isotype. These data suggest that the number of OT-I cells present before re-challenge is a key determining factor for the strength of the recall response and that mIgG1 and mlgG2a mAb deliver signals during the primary response which results in different resting memory populations.

To understand this difference in memory response OT-I T cells in the blood were phenotyped during the primary and memory stages. Analsyis of CD127 and KLRG1 expression during the primary response can identify short-lived effector cells (SLECs - CD127-KLRG1+) and memory precurosor cells (MPECs - CD127+KLRG1-) [26]. The frequency of MPECs was higher in the mlgG1 groups at Day 18 (Fig 3C). Whilst frequencies of SLECs in the blood was similar between isotypes (Supplemental Fig 4D) granzyme B production was higher in SAP 9 and 25-29 mlgG2a treated mice compared to mlgG1 treated mice (Fig. 3C). Additionally these data indicated that there may be a domain related trend in granzyme $B$ production in those mice receiving mIgG2a mAb, with the following hierarchy; CRD4 binding mAb (SAP 2529) > CRD3 (SAP 9) > CRD2 (SAP 15-3) and CRD1 (SAP 28-2). In infection models, the relative frequencies of each subpopulation (SLECs vs MPECs) in the primary response does not always correlate with the accumulation of CD8+ cells during a recall response [26-28]. We therefore expanded our analysis to CXCR3 and CD43, shown to define three distinct populations of memory cells with a hierarchy of recall response $\left(\mathrm{CXCR}^{\mathrm{hi}} \mathrm{CD} 43^{\text {lo }}>\mathrm{CXCR} 3^{\text {hi }} \mathrm{CD} 43^{\text {hi }}>\mathrm{CXCR} 3^{\text {lo }} \mathrm{CD} 43^{\text {lo }}\right)[29,30]$. Mice that had been treated with mIgG1 anti-hOX40 mAb gave rise to a higher frequency of $\mathrm{CXCR}^{\text {hi }}{ }^{\mathrm{CD}} 43^{\text {lo }}$ and $\mathrm{CXCR} 3^{\text {hi }} \mathrm{CD} 43^{\text {hi }}$ cells in comparison to mice that had been treated with migG2a mAb (Fig. 3D and Supplemental Fig. 4E). This effect was most strongly seen with the SAP 25-29 CRD4-binding antibody (Fig. 3D and Supplemental Fig. 4E). This difference in frequencies between mlgG1 and mlgG2a treated mice, whilst slight during the contraction phase (D18), became more evident during the resting memory period prior to re-challenge (D53). Immediately following rechallenge with SIINFEKL peptide (D74), both mIgG1 and mIgG2a groups displayed expansion of cells with high proliferative capacity but further into the memory 
response (D78) the higher prevelance of cells with greater proliferative capacity in mlgG1 treated mice was re-established (Fig. 3D). Furthermore, mice treated with mIgG2a anti-hOX40 mAb had a higher frequency of effector-like memory cells $\left(\mathrm{CXCR} 3^{\mathrm{lo}} \mathrm{CD} 43^{\mathrm{lo}}\right)$ in the resting memory phase (D53) (Fig 3D). Again this contrast between mlgG1 and mlgG2a treated mice was re-established from D78 following rechallenge. These data indicate, that in our OT-I transfer model, the choice of isotype significantly impacts the development of a robust memory response.

These results also highlighted a discrepancy in the effect of the mlgG2a anti-hOX40 mAb between in vitro and in vivo experiments. mlgG2a anti-hOX40 mAb caused an inhibition of proliferation in vitro (Fig. 2F), whereas T cell expansion was seen in vivo (Fig. 3B). Therefore, hOX40KI ${ }^{+/-}$CD8+ OT-I T cells were purified and transferred into WT C57BL/6 mice to see if both mlgG1 and mlgG2a isotypes were capable of acting directly on CD8+ T cells (Fig 3E). Whilst mIgG1 mAb were able to drive a similar expansion as before, mlgG2a mAb had no effect (Fig. 3F). These results indicate that the mlgG2a mAb act indirectly, through non-CD8+ T cells, to facilitate OT-I expansion in contrast to the ability of mIgG1 to act directly on the OT-I T cells.

\section{anti-hOX40 mlgG2a mAb deplete hOX40 expressing cells.}

We hypothesised that the indirect effect of mlgG2a mAb might involve deletion of Treg [24]. Depletion of Tregs has previously been shown to allow greater expansion of OT-I and Ova responses [31-33]. Therefore to address this hypothesis, spleens were harvested from hOX40 $\mathrm{KI}^{+/+}$mice four days after treatment with either antihOX40 mlgG1 or migG2a mAb (Fig. 4A). Numeration of different T cell subsets in the spleen showed that the mlgG2a mAb were able to significantly reduce the Treg population and to a lesser extent CD4+ effectors whereas mIgG1 treated mice evoked expansion of T cell populations (CD8+ and OT-I) (Fig. 4B and Supplemental Fig. 5A). The relative deletion of $T$ cell populations in the mlgG2a treated mice correlated with the amount of hOX40 surface expression seen following activation in vitro (Fig. 1C) as the most significant difference was seen in the Treg population followed by CD4+ effectors. The CD8:Treg ratio was unchanged following treatment with the mlgG1 mAb, however SAP 25-29 mlgG2a produced a significant increase in the CD8:Treg ratio a with a trend towards an increase also seen for SAP 15-3 mlgG2a and SAP 9 mlgG2a (Fig. 4B). Interestingly SAP 9 mlgG2a also showed a 
decrease in inter-sample variability when looking at OT-I cell numbers and a trend towards a reduction in CD8+ $\mathrm{T}$ cell numbers, indicating superior deletion capacity compared to other mAb. Therefore, we assessed the ability of SAP 9 to delete human Tregs in vivo. Un-activated human Treg do not typically express appreciable OX40 levels (Fig. 1D, [34]), so a NOG:PBMC transfer model was used whereby hPBMCs are first activated through a xenoresponsive graft versus host response before being transferred into a new NSG recipient mouse where deletion could then be assessed. The transferred Treg upregulate hOX40 to levels similar to those observed within tumours and significantly above the levels of CD8+ T cells (Supplemental Fig. 5B). Using this approach, SAP 9 hlgG1 was shown to be capable of deleting human Tregs at least as well as the clinically-approved Treg deleting antiCTLA-4 mAb Yervoy (Fig. 4D); thereby augmenting the CD8:Treg ratio (Fig. 4E). Furthermore, when using a humanised version of SAP 9 hlgG1, significant depletion of human Tregs was observed (Fig. 4F), alongside a significant improvement in the CD8:Treg ratio (Fig. 4G), unlike CAMPATH-1 which deleted all T cells. These data indicate that with the correct isotype and significant expression of hOX40, antihOX40 antibodies are capable of specifically depleting Tregs and improving CD8:Treg ratios in vivo.

\section{Extent of isotype activity correlates with OX40 domain specificity}

Throughout the data detailed above it became apparent that the strength of deletion/agonism was associated with the domain of hOX40 bound by the various mAb. To assess this further we grouped our results into membrane distal (CRD1+2) and membrane proximal (CRD3+4) binding mAb (Fig. 5A and B). We also assessed groups reflecting those able (CRD4) or unable to bind in the presence of ligand $(\mathrm{CRD} 1+2+3)$ to see if there was a correlation with ligand competition (Fig. $5 \mathrm{C}$ and D). The strength of agonism seen with the mlgG1 mAb was highest for membraneproximal binding mAb for both expansion of OT-I cells and Tregs (Fig. 5A). In contrast, with the mlgG2a isotype only depletion of Tregs correlated with domain, again being greatest for membrane proximal mAb (Fig. 5B). Likewise, mAb binding outside of the ligand-binding domain (i.e. CRD4) displayed the highest level of mlgG1-mediated agonism (Fig. $5 \mathrm{C}$ ). Interestingly the ability of mlgG2a mAb to to deplete Tregs did not significantly correlate with binding to CRD4 (Fig 5D). 


\section{Ability of hOX40 mAb to control tumour growth}

342 To determine the immunotherapeutic potential of the anti-hOX40 mAb in vivo in our $343 \mathrm{hOX} 40^{+/+} \mathrm{KI}$ mice, we evaluated a mAb which bound to each CRD of hOX40 as both 344 a mlgG1 and mIgG2a isotype. hOX $40^{+/+} \mathrm{KI}$ mice were inoculated with E.G7-Ova lymphoma cells and subsequently treated with anti-hOX40 mAb once tumours had established. anti-hOX40 mAb, as both mlgG1 and mlgG2a, were able to elicit tumour control, with mice eradicating established tumours in most treatment groups (Fig. $6 A)$. With the exception of SAP 25-29, the mlgG1 mAb caused a higher \% survival than the mlgG2a mAb, although there was no obvious domain preference amongst the antibodies in terms of anti-tumour activity. Importantly, mice also appeared to form durable memory responses as upon re-challenge no mice developed a secondary tumour (Supplemental Fig 6A).

To determine the mechanism of tumour control, organs from tumour bearing mice were harvested and assessed for changes in immune inflitrate. Consistent with data from the OT-I model, the general trend seen in mlgG1 treated mice was expansion of $T$ cells subsets whilst migG2a mAb caused T cell depletion (Fig. 6B and C and Supplemental Fig. 6B and C). This effect was most prominent in the spleen but also observed within the tdLN (CD4+ and Tregs). Cell numbers recovered from tumours were very small therefore it was difficult to ascertain clear trends within the TILs (Fig. 6B and C). A high CD8:Treg within human tumours is associated with prolonged survival [35] and depletion of Tregs allows for anti-tumour immunity and rejection $[36,37]$. Our results show that mice treated with mlgG2a anti-hOX40 tended to increase the CD8:Treg ratio within the spleen, tdLN, and tumour (Fig. 6C). In migG1 treated mice whilst the spleen showed a significant reduction in the CD8:Treg ratio, in the tumour an increased trend was observed (Fig. 6B). It is unclear whether this discrepancy between locations occurs as a result of different response kinetics, with priming occurring in lymphoid organs and subsequent recruitment to the tumour or perhaps direct priming within the TME whereby the greater antigen density leads to a more rapid response and hence increase in CD8:Treg. A domain trend could also be seen within some of the T cell subsets; OT-I depletion in the spleen and CD4+/Treg expansion within the tdLN associated with membrane proximal domains (Fig. 6B and C and Supplemental Fig. 6B and C). 
375 The T-box transcription factors T-bet and eomesodermin (Eomes) cooperate to 376 promote cytotoxic lymphocyte formation, which correlates with the upregulation of 377 perforin and granzyme $B$ in antigen specific cells [26, 38, 39], as well as sustaining 378 memory phenotypes [40]. Thus expression of T-bet and Eomes was examined in T 379 cells within the spleen, tdLN and tumour of anti-hOX40 treated mice to better 380 understand the mechanisms involved. Both isotypes increased the T-bet+Eomes+ 381 double positive cells within the CD8+ populations (Fig. 6D) however in CD4+ T cells, only mlgG1 increased this population and only in splenic populations

383 (Supplementary Fig 7A). A domain trend was also broadly observed in CD8+ 384 populations from mlgG1 treated mice, most obviously within the spleen and tumour. CD8+ $T$ cells within the spleen and tumour also saw an increase in granzyme $B$ producing cells in both mlgG1 and mlgG2a treated mice (Supplementary Fig. 7B). As with Tbet+Eomes+ populations, $\mathrm{CD} 4+\mathrm{GzmB}+$ cells were not significantly increased with either isotype (Supplementary Fig. 7C), thus suggesting that in this model, we do not see a significant impact on cytotoxic CD4+ T cells. However these results do show that all anti-hOX40 mAb, irrespective of isotype or domain binding region, are able to produce functional effector $C D 8+T$ cells, as well as $C D 8+T$ cells expressing transcription factors important for effector and memory cell formation within a tumour environment.

To determine if the ability of these mAb to evoke tumour control was consistent across tumour models we utilised the subcutaneous MCA-205 sarcoma. Using SAP 25-29 as a paradigm, we again saw both isotypes providing tumour control, albeit more limited in comparison to the E.G7 tumour model and with no isotype preference (Figure 6E).

\section{Discussion}

403

In the current study, we generated and characterised a panel of mAb targeting hOX40. Their ability to bind throughout the four different hOX40 CRDs and expression as both mlgG1 and mlgG2a isotypes allowed investigation of the effects of both isotype and domain binding on agonistic and therapeutic potential in a newly developed hOX40 KI mouse model. reflecting that seen on healthy hPBMCs and samples from ovarian cancer patients 
with a hierarchy of expression of Treg $>C D 4+>C D 8+$. Nevertheless, differences were observed such that higher levels of hOX40 were seen on peripheral CD8+ T cells in the homozygous KI mouse than would be expected on hPBMC. Additionally, constitutive expression of hOX40 was observed on peripheral Tregs in the mice, in contrast to negligible levels on resting hPBMCs. However, this expression pattern reflects that observed on Treg in TILS isolated from cancer patients and so appears a reasonable model for studies in oncology.

417

In the hOX40 KI mouse, mOX40L not hOX40L is present which may have led to immune defects due to the absence of OX40L:OX40 interaction. However, no overt differences in immune development or homeostasis were observed in the homozygous hOX40 KI mice. Furthermore mOX40L has been shown to make similar contacts to hOX40 as hOX40L, [21], suggesting that the OX40L:OX40 signals would be maintained in our model. It also indicates that the model is suitable to address any influences of ligand binding on the activity of the mAb panel. Only mAb binding to the most membrane proximal domain (CRD4), were able to bind in the presence of the natural ligand OX40L (CD252), raising the possibility that certain effector functions could be influenced by concurrent ligand binding for the CRD4 binding mAb. Although not studied directly, no overt effects appeared to be driven by the presence or absence of OX40L binding; i.e. all antibodies regardless of ligand blocking were able to elicit function in an OT-I transfer model and in tumour models.

It is well known that isotype helps dictate mAb effector function due in part to differences in Fc $\gamma \mathrm{R}$ interactions, and so isotype choice is important for delivering therapeutic efficacy according to the mAb mechanism of action [24, 41, 42]. For TNFR family members; mlgG1 antibodies have been agonistic with engagement of Fc $\gamma R$ II and mIgG2a being either inhibitory or with limited agonistic effects yet capable of activatory Fc $\gamma \mathrm{R}$-mediated target cell depletion [12, 18, 24, 25]. Our hPBMC proliferation data added to this evidence, with hOX40 mlgG2a mAb resulting in a decrease in the percentage of proliferating T cells whereas mlgG1 counterparts evoked increases in T cell proliferation. However this was not reflected in our hOX40/- OT-I transfer studies in hOX40 ${ }^{+/+} \mathrm{KI}$ mice, where both anti-hOX40 mAb 
OT-I cells were transferred into WT mice the mIgG2a mAb were no longer able to elicit expansion of OT-I cells, unlike the mlgG1 mAb, which retained this activity. Together, these results indicate that the mlgG1 mAb causes direct agonism on the hOX40+/- OT-I cells resulting in their expansion, whereas the mlgG2a mAb require hOX40 expressing non-CD8+ cells to provide expansion. Previous experiments by Ruby et al indicated a requirement for CD4+ T cells in the expansion of OT-I T cells via anti-mouse OX40 mAb [43]. In those experiments in MHC Class II KO mice, a reduction in the resting memory population was observed, with no significant change at the peak of the primary response as we show here with the mlgG2a. There are many differences in experimental set up, including the number of OT-I cells transferred, and site of priming, as well as the isotype used. Experiments performed by Ruby et al used the anti-mOX40 mAb OX86 which is a Rat IgG1 (rlgG1). rlgG1 interacts with only Fc $\gamma \mathrm{RIIb}$ and Fc $\gamma \mathrm{RIII}$, giving it a low activatory Fc $\gamma \mathrm{R}$ : inhibitory Fc $\gamma R$ binding $(A: I)$ ratio, in contrast to the mlgG2a which interacts strongly with all activatory $\mathrm{F} c \gamma \mathrm{R}$ and has a higher $\mathrm{A}: \mathrm{I}$ ratio [44]. Thus whilst the mlgG2a is likely to mediate its effects through depletion, based upon strong activatory Fc $\gamma \mathrm{R}$ interactions, the rlgG1 through its relatively greater interaction with Fc $\gamma \mathrm{RIIb}$ is likely to have the additional capacity of direct agonism. Hence in the MHC Class II KO mice there is the possibility for the OX86 rlgG1 to directly agonise the transferred OT-I T cells via Fc $\gamma$ RIIb mediated crosslinking, unlike the ahOX40 mlgG2a when used in WT recipients, potentially explaining the disparity between the two data sets.

Enumerating the subpopulations of T cells within the spleen of hOX $40^{+/+} \mathrm{KI}$ mice revealed that the mlgG2a anti-hOX40 mAb uniquely caused depletion of Tregs with lower levels of depletion seen in the CD4+ effector population. In contrast, the mIgG1 anti-hOX40 mAb caused expansion of all T cell populations, most significantly in the general CD8+ and OT-I populations. Assuming the presence of relevant effector cells and normal distribution of FcyRs in our OT-I model, it is likely that differential Fc $\gamma \mathrm{R}$ interactions of the different isotypes explains their disparate

472 effects. Furthermore the superior affinity for the activatory $\mathrm{Fc} \gamma \mathrm{R}$ and resultant 473 deletion of suppressive Treg cells seen in mice treated with migG2a mAb likely 474 explain the mechanism behind the expansion of OT-I cells in the blood and spleen of hOX $40^{+/+} \mathrm{KI}$ but not WT mice. 
477 Another disparity seen between the anti-hOX40 mlgG1 and mlgG2a treated mice 478 was the recall response to SIINFEKL peptide in the OT-I model. Considering the similar levels of OT-I cells at the peak of the primary response it was surprising to observe such a difference in the frequency in the memory phase. A positive correlation between the frequency of OT-I cells pre-recall and the frequency of OT-I cells at the peak of the memory response highlighted the possibility that it was simply the result of the amount of cells present at the time of re-challenge, with mIgG1 mAb but not mlgG2a mAb providing signals for survival/persistence. Within the primary response the MPEC population (CD127 ${ }^{+} \mathrm{KLRG1}^{-}$) was increased at day 18 in mice treated with mlgG1 mAb compared to those treated with mlgG2a. Those mice also generated a higher percentage of $\mathrm{CXCR} 3^{\text {hi }} \mathrm{CD} 43^{\text {lo }}$ highly proliferative cells in comparison to mice treated with the mlgG2a mAb. These findings, alongside those relating to the frequency of antigen specific cells pre-rechallenge explain the disparity in the recall response comparing mlgG1 versus mlgG2a mAb. The exact mechanisms underpinning this dichotomy are not immediately clear but one possibility is that mlgG2a deplete Tregs alongside, to a lesser extent, CD4+ effectors, to influence priming. Importantly though, this higher proportion of effector cells in mlgG2a treated mice versus highly proliferative cells in mlgG1 treated mice may explain these differences in memory but moreover provide a rationale for how both isotypes are able to cause equivalent efficacy in mouse tumour models, despite exhibiting opposing mechanisms of action.

Analysis of affinity, on- and off-rates failed to reveal a correlation with activity. However, despite the limited numbers of antibodies against each individual domain, our data suggests a correlation between domain binding and strength of both mIgG2a depletion and mIgG1 agonism. anti-hOX40 mAb which bound to CRD4 were more potent agonists as mIgG1 when compared to mAb which bound to CRD1-3, although testing against a wider panel of antibodies would be required to strengthen this finding. This directly contrasts with our previous observations with anti-CD40 mAb [16] where CRD1 binding mAb were more agonistic. However, for both the antihCD40 and anti-hOX40 mAb tested, optimal agonistic function correlated with binding outside the natural ligand binding region. Potentially suggesting that the combined effects of both the ligand and the mAb in clustering the receptor are 
510 required to elicit optimal agonism. This conjecture is partially supported by recent

511 evidence from Zhang et al. who also demonstrated strong agonistic function with a

512 CRD4 binding anti-mOX40 mAb. Those authors also documented equivalent

513 agonistic activity with a CRD2-binding, ligand blocking anti-mOX40 mAb [17],

514 indicating the fine epitope is also important, as we reported previously for anti-CD40

515 [16].

516

517 The ability of TNFR targeting mAb to cause depletion of intratumoral Tregs has been

518 clearly demonstrated in recent years [8, 12, 45, 46], both increasing their therapeutic

519 possibilities but confusing potential mechanisms of action. To assess, in a more

520 translational setting, the depleting ability of our anti-hOX40 mAb, we performed

521 depletion experiments in NSG mice engrafted with human target cells. In these

522 experiments, hOX40 and other TNFR family members become upregulated on the

523 activated Treg (Supplemental Fig. 5B and [13]), enabling them to serve as targets

524 akin to those seen in tumour samples. When compared with the clinically relevant

525 Treg depletor Yervoy [47], SAP 9 hlgG1 was not only as strong a Treg depleter but

526 also generated a higher CD8:Treg ratio indicating the therapeutic potential of this

527 anti-hOX40 mAb. Importantly, deletion of Treg was specific even though all T cell

528 subsets had expanded and were activated through the NSG passage.

529

530 Having established the agonistic and depletory capacity of our mAb, it was then

531 perhaps slightly surprising for both isotypes to act therapeutically to a similar extent

532 in tumour models but likely reflects their relative propensity to elicit different effector

533 functions in the tumour microenvironment (TME). The latter is known to have

534 profound effects on therapeutic efficacy [48]. A tumour with a high infiltrate of cells

535 expressing activatory Fc $\gamma \mathrm{R}$ such as NK cells and macrophages, is likely to be more

536 responsive to a mlgG2a mAb and depletion of detrimental target cells than a T cell-

537 agonising mlgG1 mAb. Conversely, if the inhibitory Fc $\gamma \mathrm{R} I \mathrm{BB}$ is more prevalent,

538 mlgG1-mediated agonism may be more prominent. Lymphoid organs outside the

539 tumour may also be relevant. In the E.G7-Ova model, although the magnitude of

540 effect differed, the mlgG1 mAb largely caused T cell expansion and the mIgG2a

541 depletion in spleen and draining lymph nodes. It seems likely therefore that these

542 intrinsic differences underpin the relevant mechanism of action in each case. 
543 Accordingly, mlgG2a reagents likely achieve therapeutic effects through depleting

544 Tregs, releasing T cell effector responses whereas mlgG1 expand T cell numbers 545 and hence increasing cytotoxic effectors within the tumour site. Despite the disparity 546 in recall responses in the OT-I model (mlgG1>>mlgG2a), in the E.G7-Ova model

547 both isotypes were able to elicit tumour control and generate memory sufficient to 548 prevent tumour growth upon rechallenge. This suggests that other factors must be 549 operational in the presence of tumour. One possibility is that the threshold for 550 memory is relatively low in the presence of an immunogeneic tumour and that the 551 limited level of memory recall seen following treatment with the mlgG2a is sufficient.

552

553

554

555

556

557

558

559

560

561

562

563

564

565

566

567

568

569

570

571

572

573

574

575 Alternatively, it may indicate that the mlgG2a mechanism of action, deleting Tregs, allows the expansion of otherwise cryptic epitopes allowing $\mathrm{T}$ cell control as was previously described in the CT26 model [49].

In recent years, evidence for cytotoxic CD4+ T cells in tumour eradication has been reported $[50,51]$. Through the use of both adoptive transfer models and the B16 F10 melanoma model, Qui et al showed that OX40 mAb stimulation increased GzmB production in CD4+ T cells and that dual co-stimulation with 4-1BB could expand these cells [51]. In our E.G7 model however, we failed to see consistent increases in either CD4+ GzmB+ T cells or CD4+ Eomes+Tbet+ T cells in response to anti-OX40 mAb (Supplementary Fig 7 A-C) regardless of isotype used. This potentially reflects the differences in how GzmB was measured in the two tumour models as in the B16 F10 model, isolated cells were stimulated for 24 hours with anti-CD3 mAb prior to GzmB detection. As we measured GzmB directly ex vivo we cannot rule out that upon restimulation we would also have revealed CD4+ cytotoxic potential in response to OX40 stimulation.

In summary, our findings show that immunomodulatory mAb directed against hOX40 can harness multiple mechanisms of action to elicit tumour control. They also show that these mechanisms can be modulated dependent upon the choice of isotype and domain binding region. Targeting the membrane proximal domains appears optimal for both deletion and agonism; with the latter strongly driven by isotypes with low A:I ratios such as $\mathrm{mlgG1}$. Lowering the $A: I$ ratio can be attained in many different ways, for example by increasing the affinity for Fc $\gamma$ RIIB, which has been shown to mediate 
576 more effective agonism for anti-CD40 mAb [52] and a recent paper showed that this 577 may also be true for OX40 using a clinically relevant antibody [53] in vitro but as yet 578 it is unclear whether this will be true in vivo for anti-hOX40 mAb. These findings have 579 implications for the design of the next generation of anti-hOX40 mAb for the clinic. 580 Current reagents typically display an unmodified hlgG1 isotype and although safe 581 have not delivered strong anti-tumour effects [6]. These hlgG1 reagents would be 582 expected to deliver the Treg deleting function indicated here but to date this activity 583 has not been shown clearly in patients. Furthermore, their deletion may not be sufficient to elicit tumour regression in most human cancers, unlike the mouse models shown here. Therefore, mAb with appropriate isotypes and further engineering (hlgG2B[16, 54], SELF[52, 55], V11[52, 56]) to elicit potent T cell agonism may be warranted for further investigation for use both as a monotherapy but more importantly in combination.

\section{Methods and Materials}

Human Samples

593

Peripheral blood mononuclear cells (PBMCs) were obtained from healthy adult volunteers from either Southampton National Blood Service, UK or Hallands Hospital Halmstad, Sweden. For NSG reconstitution experiments performed in Southampton, hPBMCs were purchased from STEMCELL Technologies.

Mice

C57BL/6 mice and OT-I transgenic mice were obtained from Charles River

600 Laboratories. NSG mice were purchased from Jackson Laboratories. hOX40 knockin mice were generated by Ozgene. hOX40/OT-I mice were generated in house. For all experiments young adult mice were sex- and age-matched and randomly assigned to experimental groups. Experiments were not blinded.

604

Antibody Production and labelling Anti-hOX40 mAb were produced and labelled using standard techniques as detailed in supplementary methods. Cetuximab was a kind gift from Thomas Valerius and

608 Campath-1 was a kind gift from Geoff Hale. 
611 The variable regions of SAP 9 heavy and light chains were sequenced from the

612 hybridoma by PCR. The sequence was humanised using Macromoltek's proprietary

613 humanisation algorithms. A generic antibody signal peptide sequence was then

614 added to the humanised variable region sequences and the amino acid sequences

615 converted into nucleotide sequences using

616 https://www.bioinformatics.org/sms2/rev_trans.html. Nucleotide sequences were

617 synthesized by GeneArt and subcloned into expression vector pEE6.4 (Lonza) for

618 expression.

619

620 Binding domain determination/ blocking experiments

621 hOX40 constructs were transiently transfected into 293F cells before addition of

$62210 \mu \mathrm{g} / \mathrm{ml}$ anti-hOX40 mAb, binding was detected with a PE-labelled secondary anti-

623 mouse Fc antibody (Jackson Laboratories). For blocking experiments unlabelled

624 antibody was added for 30 minutes before addition of FITC-labelled anti-hOX40

625 mAb. Binding was assessed using flow cytometry (see below).

626

627 Surface Plasmon Resonance

628 A Biacore T100 upgraded to a T200 (GE Life Sciences) was used to measure

629 interactions with hOX40. 100nM of hOX40L-His was immobilised onto a CM5 chip

630 (GE Healthcare) coated with an anti-His mAb. 100nM hOX40-hFc was subsequently

631 captured followed by the injection of anti-hOX40 mAb $(15 \mu \mathrm{g} / \mathrm{ml})$.

632

633 In vitro assays

634 Standard hPBMC proliferation assays were performed as detailed in supplementary

635 methods. For expression assays frozen hPBMCs were thawed and rested overnight,

636 then stimulated with plate bound anti-CD3 (OKT3, 15ng/ml) and soluble anti-CD28

637 (CD28.2, 0.5 $\mu \mathrm{g} / \mathrm{ml})$. Cells were harvested, stained with appropriate antibodies and

638 assessed via flow cytometry.

639 Murine expression assays; standard activation was used as detailed in

640 supplementary methods. Cells were stained with appropriate antibodies and

641 analysed by flow cytometry.

642

643 Flow cytometry 
Standard staining and analysis performed as detailed in supplementary methods.

645

646

OT-I Adoptive Transfer

647 $1 \times 10^{5} \mathrm{hOX} 40 \mathrm{KI}^{+/-}$OT-I cells were injected i.v. into hOX40 $\mathrm{KI}^{+/+}$or WT C57BL/6 mice. 24 hours later 5mg ovalbumin (Sigma) and 100 $\mathrm{gg}$ anti-hOX40 or isotype control were given i.p. Deletion was determined by harvesting spleens day 4 post i.p. injection. OT-I kinetics were monitored in the blood through SIINFEKL tetramer staining and mice were rechallenged between 6-10 weeks later with 30nM SIINFEKL i.v. Based on preliminary experiments $n=3$ was determined as sufficient to see a p $<0.05$ for OT-I expansion. Mice with SIINFEKL tetramer responses less than $1 \%$ of CD8+ lymphocytes at the peak of the response were excluded due to the likelihood that the OT-I transfer had failed since isotype controls peak at an average $5.2 \%+/$ 0.58 s.e.m. (mlgG1) and $4.65 \%+/-0.65$ s.e.m. (mlgG2a) in blood and $3.8 \%+/-0.79$ s.e.m (mlgG1) and 3.1\% +/-0.89 on Day 4 in spleens. One mouse was excluded from Figure 4 B groups SAP-28, 15-3 and 9 based on this criteria.

659

660

Treg cell depletion in reconstituted NOG/SCID mice PBMC-NOG/SCID mice (primary human xenograft model) were generated by

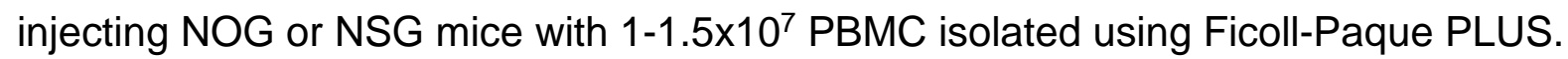
Approximately two weeks after reconstitution of NOG or NSG mice with hPBMCs, spleens were harvested. $10 \times 10^{6}$ splenocytes were then injected into the peritoneal cavity of naive SCID or NSG mice 1 hour prior to injection with $10 \mathrm{mg} / \mathrm{kg}$ of depleting mAb or isotype control mAb. The peritoneal fluid was collected after 24 hours and human $\mathrm{T}$ cell subsets were identified by flow cytometry.

Tumour Models

670 E.G7-Ova and MCA-205 tumour models: $5 \times 10^{5}$ tumour cells were injected subcutaneously into the flank of mice. Based on preliminary experiments $n=5$ was determined as sufficient to see a p<0.05 for tumour therapy. Groups of 8 (E.G7-Ova) or 6 (MCA-205) mice were set up to ensure that at treatment there would be a

674 minimum of 5 per group with established tumours with comparable size (between

$6755 \times 5$ and $8 \times 8 \mathrm{~mm}$ ). Mice were then ranked according to tumour size and assigned to treatments groups so that average tumour size per group was similar prior to 
677 treatment. This ensured mixed treatment groups within cages to reduce influence of

678 housing on treatment effect. Established tumours were treated with $3 \times 100 \mu \mathrm{g}$ anti-

679 hOX40 mAb or isotype i.p. every other day. For phenotyping experiments organs

680 were harvested day 4 post final injection. Tumours were digested using 0.5 Units of

681 liberase TL (Roche) and cells analysed via flow cytometry. For survival experiments

682 tumour size mice culled once they reached a terminal size (E.G7: 20x20mm, MCA-

683 205: 15x15mm). Mice which erradicated tumour after treatment were rechallenged

684

with $5 \times 10^{5}$ tumour cells s.c. into the flank.

685

686 Statistics

687 All results show mean \pm SEM. One way Annova with multiple comparisons

688 (Dunnett's, Tukey's or Sidak's as stated in legend) or Mann Whitney tests were used 689 as stated in legends performed using GraphPad Prism. Survival curves evaluated 690 using a Log-rank (Mantel-Cox) test. Significance shown relative to isotype control 691

692 unless bar is shown. Where indicated ns $=$ not significant, ${ }^{*} P \leq 0.05,{ }^{* *} P \leq 0.01,{ }^{* * *} P$ $\leq 0.001,{ }^{* * * *} \mathrm{P} \leq 0.0001$.

693

\section{Declarations}

695 Ethics Approval

696 All procedures were conducted in accordance with UK Home Office guidelines and were approved by the University of Southampton's ethical committee or at Biolnvent under Dnr 14760/2016. For clinical samples, ethical approval was obtained from the Ethics Committee of Skåne University Hospital, Sweden.

700

\section{Availability of Data and Material}

702 All datasets used and/or analysed during the current study are available from the corresponding author on reasonable request.

704

705

\section{Acknowledgements}

We are grateful to the staff of the University of Southampton Biomedical Research facility for their technical support. We also thank Leon Douglas and Patrick Duriez from the ECMC/CRUK funded Protein Core Facility for making SIINFEKL tetramers 
709

710

711

712

713

714

715

716

717

718

719

720

721

722

723

724

725

726

727

728

729

730

731

732

733

734

735

736

737

738

739

740

741

742

743

744

745

746

747

748

\section{Author contributions}

JG, KH, HLS, KC, RRF, HTC, TS, MS, LM and JW performed experiments. JG, KH, HLS, TS, RRF, MS, LM and JW performed statistical analyses. JG, JW, IT, BF and MSC designed experiments. JG, JW and MSC wrote the manuscript. All authors contributed to manuscript revision and read and approved the submitted version.

\section{Funding}

This work was supported by CRUK programme grants awarded to MJG and MSC (Award number: A20537, A24721), CRUK centre grant (Award number: A25139) and a CRUK studentship to JG and MSC from the Southampton CRUK centre (Award number: A29286).

\section{Conflict of interest}

MSC is a retained consultant for Biolnvent International and has performed educational and advisory roles for Baxalta and Boehringer Ingleheim. He has received research funding from Roche, Gilead, Bioinvent International and GSK. BF, IT, LM and MS are employees of Bioinvent International.

\section{References:}

1. Seidel, J.A., A. Otsuka, and K. Kabashima, Anti-PD-1 and Anti-CTLA-4 Therapies in Cancer: Mechanisms of Action, Efficacy, and Limitations. Front Oncol, 2018. 8: p. 86.

2. Dine, J., et al., Immune Checkpoint Inhibitors: An Innovation in Immunotherapy for the Treatment and Management of Patients with Cancer. Asia Pac J Oncol Nurs, 2017. 4(2): p. 127-135.

3. Remer, M., et al., The Use of Anti-CD40 mAb in Cancer. Curr Top Microbiol Immunol, 2017. 405: p. 165-207.

4. Bartkowiak, T. and M.A. Curran, 4-1BB Agonists: Multi-Potent Potentiators of Tumor Immunity. Front Oncol, 2015. 5: p. 117.

5. Aspeslagh, S., et al., Rationale for anti-OX40 cancer immunotherapy. Eur J Cancer, 2016. 52: p. 50-66.

6. Buchan, S.L., A. Rogel, and A. Al-Shamkhani, The immunobiology of CD27 and OX40 and their potential as targets for cancer immunotherapy. Blood, 2018. 131(1): p. 3948.

7. Kuang, Z., et al., Development and characterization of a novel anti-OX40 antibody for potent immune activation. Cancer Immunol Immunother, 2020.

8. $\quad$ Freeman, Z.T., et al., A conserved intratumoral regulatory $T$ cell signature identifies 4$1 B B$ as a pan-cancer target. J Clin Invest, 2020. 130(3): p. 1405-1416.

9. Croft, M., Costimulation of T cells by OX40, 4-1BB, and CD27. Cytokine Growth Factor Rev, 2003. 14(3-4): p. 265-73. 
10. Li, F. and J.V. Ravetch, A general requirement for FcgammaRIIB co-engagement of agonistic anti-TNFR antibodies. Cell Cycle, 2012. 11(18): p. 3343-4.

11. White, A.L., et al., FcgammaRlotalotaB controls the potency of agonistic anti-TNFR mAbs. Cancer Immunol Immunother, 2013. 62(5): p. 941-8.

12. Bulliard, Y., et al., OX40 engagement depletes intratumoral Tregs via activating FcgammaRs, leading to antitumor efficacy. Immunol Cell Biol, 2014. 92(6): p. 475-80.

13. Buchan, S.L., et al., Antibodies to Costimulatory Receptor 4-1BB Enhance Anti-tumor Immunity via T Regulatory Cell Depletion and Promotion of CD8 T Cell Effector Function. Immunity, 2018. 49(5): p. 958-970 e7.

14. Weinberg, A.D., et al., Science gone translational: the OX40 agonist story. Immunol Rev, 2011. 244(1): p. 218-31.

15. Cleary, K.L.S., et al., Antibody Distance from the Cell Membrane Regulates Antibody Effector Mechanisms. J Immunol, 2017. 198(10): p. 3999-4011.

16. $\mathrm{Yu}, \mathrm{X}$., et al., Complex Interplay between Epitope Specificity and Isotype Dictates the Biological Activity of Anti-human CD40 Antibodies. Cancer Cell, 2018. 33(4): p. 664675 e4.

17. Zhang, P., et al., Ligand-Blocking and Membrane-Proximal Domain Targeting AntiOX40 Antibodies Mediate Potent T Cell-Stimulatory and Anti-Tumor Activity. Cell Rep, 2019. 27(11): p. 3117-3123 e5.

18. Bulliard, Y., et al., Activating Fc gamma receptors contribute to the antitumor activities of immunoregulatory receptor-targeting antibodies. J Exp Med, 2013. 210(9): p. 1685-93.

19. Fujita, T., et al., Functional characterization of OX40 expressed on human CD8+ $T$ cells. Immunol Lett, 2006. 106(1): p. 27-33.

20. Xie, F., et al., Characterization and application of two novel monoclonal antibodies against human OX40: costimulation of T cells and expression on tumor as well as normal gland tissues. Tissue Antigens, 2006. 67(4): p. 307-17.

21. Compaan, D.M. and S.G. Hymowitz, The crystal structure of the costimulatory OX4OOX4OL complex. Structure, 2006. 14(8): p. 1321-30.

22. Willoughby, J., et al., OX40: Structure and function - What questions remain? Mol Immunol, 2017. 83: p. 13-22.

23. Croft, M., Control of immunity by the TNFR-related molecule OX4O (CD134). Annu Rev Immunol, 2010. 28: p. 57-78.

24. Beers, S.A., M.J. Glennie, and A.L. White, Influence of immunoglobulin isotype on therapeutic antibody function. Blood, 2016. 127(9): p. 1097-101.

25. Croft, M., C.A. Benedict, and C.F. Ware, Clinical targeting of the TNF and TNFR superfamilies. Nat Rev Drug Discov, 2013. 12(2): p. 147-68.

26. Joshi, N.S., et al., Inflammation directs memory precursor and short-lived effector $C D 8(+) T$ cell fates via the graded expression of T-bet transcription factor. Immunity, 2007. 27(2): p. 281-95.

27. Bjorkdahl, O., et al., Characterization of CC-chemokine receptor 7 expression on murine T cells in lymphoid tissues. Immunology, 2003. 110(2): p. 170-9.

28. Kapasi, Z.F., et al., Defective generation but normal maintenance of memory $T$ cells in old mice. Eur J Immunol, 2002. 32(6): p. 1567-73.

29. Hikono, H., et al., Activation phenotype, rather than central-or effector-memory phenotype, predicts the recall efficacy of memory CD8+ T cells. J Exp Med, 2007. 204(7): p. 1625-36. 
30. Olson, J.A., et al., Effector-like CD8(+) $T$ cells in the memory population mediate potent protective immunity. Immunity, 2013. 38(6): p. 1250-60.

31. Ataera, $\mathrm{H}$., et al., The control of $C D 8+T$ cell responses is preserved in perforindeficient mice and released by depletion of $C D 4+C D 25+$ regulatory $T$ cells. J Leukoc Biol, 2013. 94(4): p. 825-33.

32. Heit, A., et al., Circumvention of regulatory CD4(+) T cell activity during cross-priming strongly enhances T cell-mediated immunity. Eur J Immunol, 2008. 38(6): p. 1585-97.

33. McNally, A., et al., CD4+CD25+ regulatory $T$ cells control CD8+ T-cell effector differentiation by modulating IL-2 homeostasis. Proc Natl Acad Sci U S A, 2011. 108(18): p. 7529-34.

34. Issa, F., et al., Transiently Activated Human Regulatory T Cells Upregulate BCL-XL Expression and Acquire a Functional Advantage in vivo. Front Immunol, 2019. 10: p. 889.

35. Sato, E., et al., Intraepithelial CD8+ tumor-infiltrating lymphocytes and a high $C D 8+/$ regulatory $T$ cell ratio are associated with favorable prognosis in ovarian cancer. Proc Natl Acad Sci U S A, 2005. 102(51): p. 18538-43.

36. Shimizu, J., S. Yamazaki, and S. Sakaguchi, Induction of tumor immunity by removing CD25+CD4+ T cells: a common basis between tumor immunity and autoimmunity. J Immunol, 1999. 163(10): p. 5211-8.

37. Onizuka, S., et al., Tumor rejection by in vivo administration of anti-CD25 (interleukin2 receptor alpha) monoclonal antibody. Cancer Res, 1999. 59(13): p. 3128-33.

38. Intlekofer, A.M., et al., Effector and memory CD8+ T cell fate coupled by $T$-bet and eomesodermin. Nat Immunol, 2005. 6(12): p. 1236-44.

39. Pearce, E.L., et al., Control of effector CD8+ T cell function by the transcription factor Eomesodermin. Science, 2003. 302(5647): p. 1041-3.

40. Banerjee, A., et al., Cutting edge: The transcription factor eomesodermin enables CD8+ T cells to compete for the memory cell niche. J Immunol, 2010. 185(9): p. 498892.

41. Bruhns, P., Properties of mouse and human IgG receptors and their contribution to disease models. Blood, 2012. 119(24): p. 5640-9.

42. Stewart, R., et al., The role of Fc gamma receptors in the activity of immunomodulatory antibodies for cancer. Journal for ImmunoTherapy of Cancer, 2014. 2(1): p. 29.

43. Ruby, C.E., et al., Anti-OX40 stimulation in vivo enhances CD8+ memory $T$ cell survival and significantly increases recall responses. Eur J Immunol, 2007. 37(1): p. 157-66.

44. Arce Vargas, F., et al., Fc-Optimized Anti-CD25 Depletes Tumor-Infiltrating Regulatory $T$ Cells and Synergizes with PD-1 Blockade to Eradicate Established Tumors. Immunity, 2017. 46(4): p. 577-586.

45. Marabelle, A., et al., Depleting tumor-specific Tregs at a single site eradicates disseminated tumors. J Clin Invest, 2013. 123(6): p. 2447-63.

46. Simpson, T.R., et al., Fc-dependent depletion of tumor-infiltrating regulatory $T$ cells co-defines the efficacy of anti-CTLA-4 therapy against melanoma. J Exp Med, 2013. 210(9): p. 1695-710.

47. Arce Vargas, F., et al., Fc Effector Function Contributes to the Activity of Human AntiCTLA-4 Antibodies. Cancer Cell, 2018. 33(4): p. 649-663 e4.

48. Klemm, F. and J.A. Joyce, Microenvironmental regulation of therapeutic response in cancer. Trends Cell Biol, 2015. 25(4): p. 198-213. 
49. James, E., et al., Differential suppression of tumor-specific CD8+ $T$ cells by regulatory $T$ cells. J Immunol, 2010. 185(9): p. 5048-55.

50. Quezada, S.A., et al., Tumor-reactive CD4(+) T cells develop cytotoxic activity and eradicate large established melanoma after transfer into lymphopenic hosts. J Exp Med, 2010. 207(3): p. 637-50.

51. Qui, H.Z., et al., CD134 plus CD137 dual costimulation induces Eomesodermin in CD4 $T$ cells to program cytotoxic Th1 differentiation. J Immunol, 2011. 187(7): p. 3555-64.

52. Dahan, R., et al., Therapeutic Activity of Agonistic, Human Anti-CD40 Monoclonal Antibodies Requires Selective FcgammaR Engagement. Cancer Cell, 2016. 29(6): p. 820-831.

53. Campos Carrascosa, L., et al., FcgammaRIIB engagement drives agonistic activity of Fc-engineered alphaOX40 antibody to stimulate human tumor-infiltrating $T$ cells. J Immunother Cancer, 2020. 8(2).

54. White, A.L., et al., Conformation of the human immunoglobulin $\mathrm{G} 2$ hinge imparts superagonistic properties to immunostimulatory anticancer antibodies. Cancer Cell, 2015. 27(1): p. 138-48.

55. Chu, S.Y., et al., Inhibition of $B$ cell receptor-mediated activation of primary human $B$ cells by coengagement of CD19 and FcgammaRIlb with Fc-engineered antibodies. Mol Immunol, 2008. 45(15): p. 3926-33.

56. Mimoto, F., et al., Engineered antibody Fc variant with selectively enhanced FcgammaRllb binding over both FcgammaRlla(R131) and FcgammaRlla(H131). Protein Eng Des Sel, 2013. 26(10): p. 589-98.

\section{Figure Legends:}

Figure 1. hOX40KI mice express hOX40 in a hierachial manner. A. Expression of OX40 (mouse $(\mathrm{m})$ and human $(\mathrm{h})$ - blue line) compared with isotype control (shaded histogram) on Treg (top row), CD4+ Effectors (middle row) and CD8+ T cells (bottom row). Representative plots shown. B Heat map summarising OX40 expression as a percentage on resting mouse splenocytes $(n=4)$. C. Expression of mOX40 (left panel) and hOX40 (right panel) on splenocytes from WT or hOX40KI mice activated with $\alpha$ CD3 and $\alpha$ CD28 $(n=3)$. Isotype controls showed as dashed lines. $D$. Expression of hOX40 on hPBMCs activated with $\alpha \mathrm{CD} 3$ and $\alpha \mathrm{CD} 28$. Histograms show hOX40 expression on Tregs (blue line, isotype control black line), CD4+ (red line, isotype control black dashed line) and CD8+ (green line, isotype control grey filled histogram) from a representative donor on Day 0 (top panel) and Day 1 (bottom panel). Line graph (right panel) shows average expression, isotype controls shown as dashed lines ( $n=3)$. E. hOX40 expression on CD4+ effector T cells (white bars), CD8+ T cells (hatched bars) or Tregs (black bars) isolated from healthy 
donors or blood, ascites and tumour from cancer patients ( $n=4-16)$. Mean +/-SEM ${ }^{\star \star \star \star *} p<0.0001,{ }^{* \star} p<0.01,{ }^{*} p<0.05$ Tukey's multiple comparison test.

Figure 2. Characterisation of a panel of anti-hOX40 mAb. A. Schematic of the WT and domain mutant hOX40 constructs generated. CRD3 from mOX40 was used to stabilise the human CRD4 construct. B. Binding of anti-hOX40 mAb to domain constructs detected by a PE labelled secondary $\mathrm{Fab}_{2}$ fragments. Representative histograms show hOX40 mAb binding (dark grey histogram) in comparison to an isotype control (light grey histogram). C. Representative histograms show antihOX40 FITC labelled mAb binding (dark grey histogram) in relation to an isotype control (light grey histogram) after binding of unlabelled anti-hOX40 mAb. The heatmap shows MFI of FITC labelled antibody binding in the presence of unlabelled antibodies with the absence of colour indicating blocking. D. Diagram summarising antibody binding domains in relation to the crystal structure of the OX40:OX40L complex. E. SPR analysis of anti-hOX40 mAb binding to hOX40-hFc in the presence of hOX40L-His fusion protein. F.Proliferation of hCD8+ T cells within PBMC cultures in response to sub-optimal anti-CD3 and anti-hOX40 mAb stimulation (representative of 4 individual donors). Mean + - SEM ${ }^{*} p<0.01,{ }^{*} p<0.05$ Dunnett's multiple comparison test.

Figure 3. anti-hOX40 mlgG1 act agonistically in vivo. A. Schematic of the OT-I model used in B-D. $1 \times 10^{5}$ hOX40KI ${ }^{+/-}$OT-I cells were transferred into hOX40KI ${ }^{+/+}$ recipients. Mice were challenged with $5 \mathrm{mg}$ Ova and $100 \mu \mathrm{g}$ of antibody. B. Kinetic analysis of OT-I expansion in response to anti-hOX40 mlgG1 mAb (left panel) or mlgG2a mAb (right panel) ( $n=4$ representative of 2 independent experiments). $C$ Analysis of memory and effector phenotyping of OT-I+ T cells in blood at Day 18. MPECs - CD127+KLRG1- (left panel) and Granzyme B+ OT-I T cells (right panel) ( $n=4$ isotype controls and $n=8$ all treatment groups, pooled from 2 independent experiments). D. CXCR3/CD43 profiling of OT-I T cells in blood at timepoints indicated in response to SAP 25-29 stimulation $(n=4)$. E. Schematic of the OT-I model used in F. $1 \times 10^{5} \mathrm{hOX} \mathrm{KKI}^{+/-}$OT-I were transferred into WT C57BL/6 recipients. Mice were challenged with $5 \mathrm{mg}$ Ova and $100 \mu \mathrm{g}$ of antibody. F. Kinetic analysis of OT-I expansion in response to anti-hOX40 mlgG1 mAb (left panel) or 
mlgG2a mAb (right panel) ( $\mathrm{n}=4$ representative of 2 independent experiments). Mean

$917+/-$ SEM ${ }^{* * *} p<0.0001,{ }^{* \star *} p<0.001,{ }^{* *} p<0.01 * p<0.05$ Sidak's multiple comparison 918 test.

920 Figure 4. anti-hOX40 mlgG2a mAb elicit Treg cell depletion in vivo. A. Schematic of

921 OT-I model used to assess cell depletion. B. Assesment of Tetramer+ve (left panel),

922 Treg cell numbers (middle panel) and CD8:Treg ratio (right panel) in response to

923 anti-hOX40mAb either as a mlgG1 (top row) or mlgG2a (bottom row) (n=4 SAP 28-

$9242, n=5$ SAP 15-3 and SAP 9, n=6 all remaining groups, pooled from two independent

925 experiments). C. Schematic of the NSG/PBMC model to assess depletion of

926 hPBMCs. $1.5 \times 10^{7}(\mathrm{D}, \mathrm{E})$ or $1 \times 10^{7}(\mathrm{~F}, \mathrm{G})$ hPBMC were transferred into NOG (D, E)

927 or NSG $(F, G)$ mice. 2 weeks later splenocytes were harvested and transferred into

928 SCID (D,E; $n=11$ SAP-9, n=12 Yervoy, $n=13$ isotype control pooled from 2

929 independent experiments) or NSG (F, G; $n=7$ pooled from 2 independent

930 experiments) recipients which were then treated with depleting antibodies. Treg

931 numbers $(D, F)$ and CD8:Treg ratio $(E, G)$ were determined by flow cytometry. Mean

$932+/-$ SEM, ${ }^{* \star \star *} p<0.0001,{ }^{* \star *} p<0.001,{ }^{* *} p<0.01,{ }^{*} p<0.05$ Sidak's (B) and Tukey's (F

933 and $\mathrm{G}$ ) multiple comparison test.

934

935 Figure 5. Anti-hOX40 mAb agonism and depleting activity are related to domain

936 binding. Model used as in Fig 4A and B with spleens harvested on Day 4 post

937 treatment. A. Analysis of OT-I cell numbers (left panels) and Treg cell numbers (right

938 panels) in response to anti-hOX40 mlgG1 mAb grouped into proximal binders -

939 CRD3+4 or distal binders - CRD1+2. B. As in A except mlgG2a mAb was used. C.

940 Analysis of OT-I cell numbers (left panels) and Treg cell numbers (right panels) in

941 response to anti-hOX40 mlgG1 mAb grouped into those which can bind in the

942 presence of ligand - CRD4 vs those which cannot - CRD1-3. D. As in C except

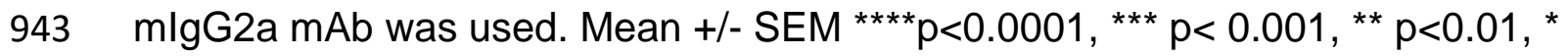

$944 p<0.05$ Tukey's multiple comparison test (A and $B ; n=6-16)(C$ and $D ; n=6-19)$.

946 Figure 6. anti-hOX40 mAb are therapeutic as both mlgG1 and mlgG2a. A. Survival

947 curves for mice challenged with E.G7 lymphoma cells $\left(0.5 \times 10^{6}\right)$ and treated with

$9483 \times 100 u g$ mAb once tumours are between $5 \times 5$ and $10 \times 10 \mathrm{~mm}$. Data pooled from 2

949 independent experiments ( $n=5$ SAP 28-2, $n=10$ SAP 15-3 and SAP 9, n=20 isotype 
950 controls and SAP 25-29). Assessment of T cell populations in spleen (top panels),

951 Tumour draining lymph node (middle panels), and Tumour (bottom panels) isolated

95224 hours post second mAb dose either as a mlgG1 (B) or mlgG2a (C). $n=5$ except

953 for SAP 28-2 mlgG2a $n=4$ for all organs and SAP15-3 and SAP 25-29 mlgG1 in

954 tumour $n=3$ due to tumour regression, representative of 2 independent experiments.

955 D. Analysis of Tbet and Eomes expression in CD8+T cells isolated from Spleen (left

956 panels), Tumour draining lymph node (middle panels) and Tumour (right panels)

957 from mice treated with either mlgG1 (top row) or mlgG2a (bottom row). Data pooled

958 from 2 independent experiments ( $n=8$ SAP 28-2, all other groups $n=9$ ). E. Survival

959 graphs for mice challenged with MCA-205 cells $\left(0.5 \times 10^{6}\right)$ and treated with $3 \times 100 u g$

$960 \mathrm{mAb}$ once tumours were $5 \times 5 \mathrm{~mm}$. Data pooled from 3 independent experiments

961 ( $n=12$ isotype mlgG1, $n=15$ isotype mlgG2a, $n=18$ SAP 25-29 mlgG2a and $n=21$

962 SAP 25-29 mlgG2a). Mean +/- SEM ${ }^{* * * *} p<0.0001$, ${ }^{* \star *} p<0.001,{ }^{* *} p<0.01,{ }^{*} p<0.05$

963 Log-rank (Mantel-Cox) for survival graphs A and E and Dunnett's multiple

964 comparison test for B-D. 
OX40 stain

mouse

mouse

human

human

Treg
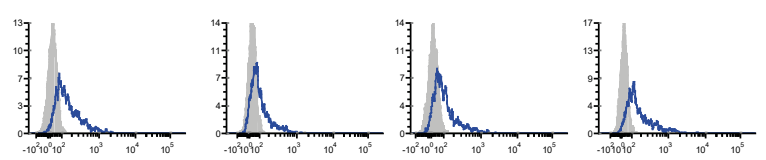

CD4

Effectors
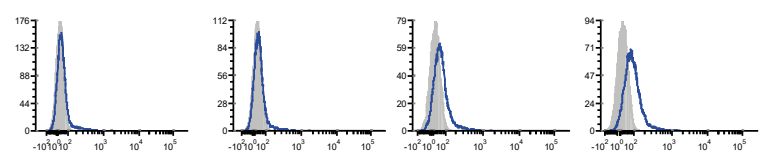

CD8+

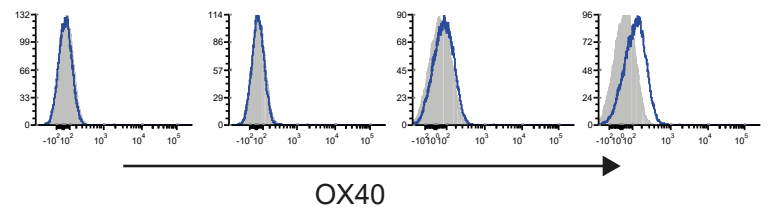

B

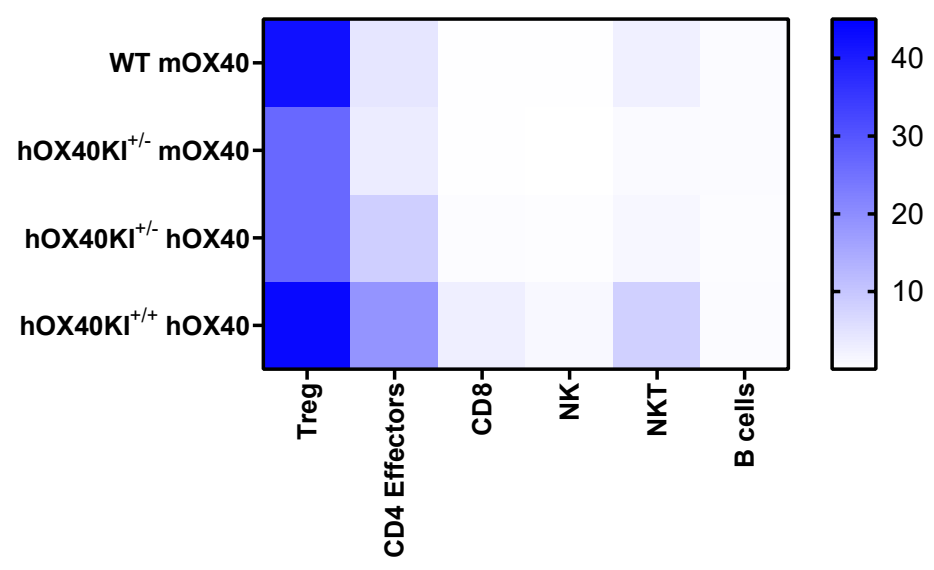

C
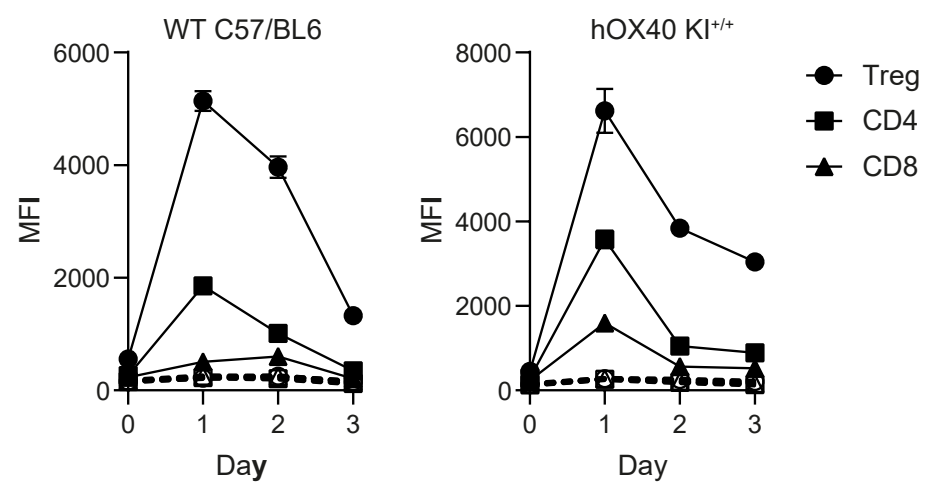

D
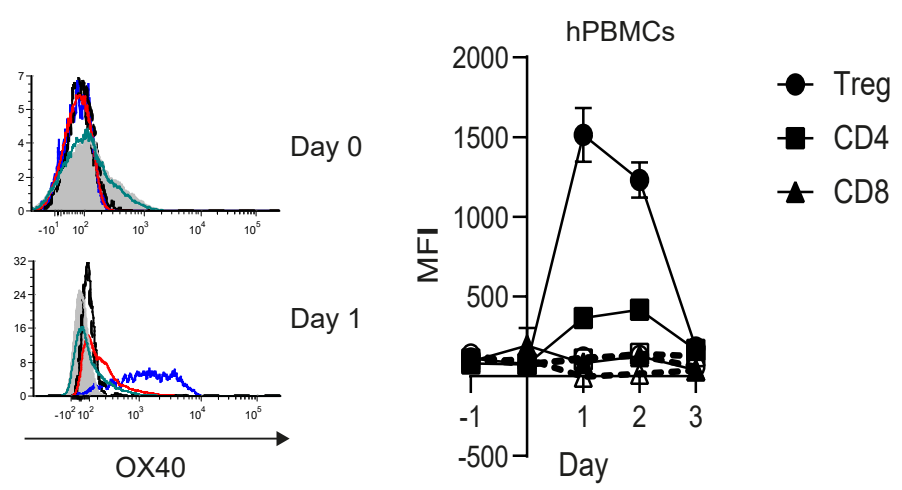

E

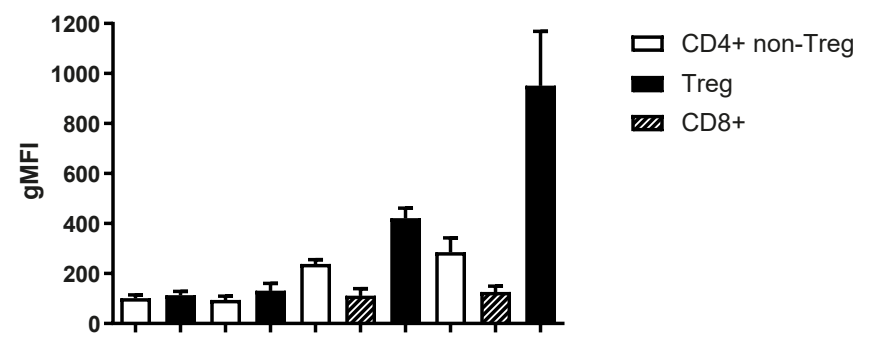


A.
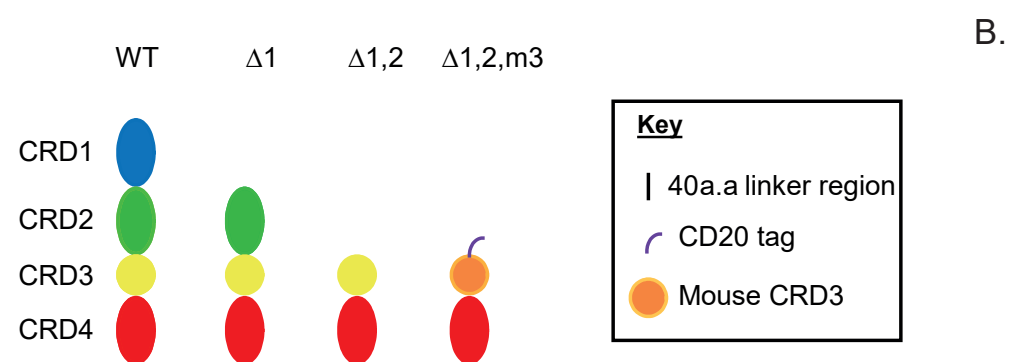

C.

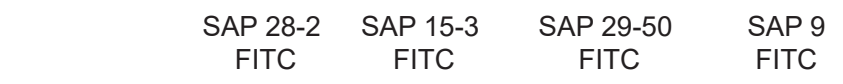

SAP 28-3
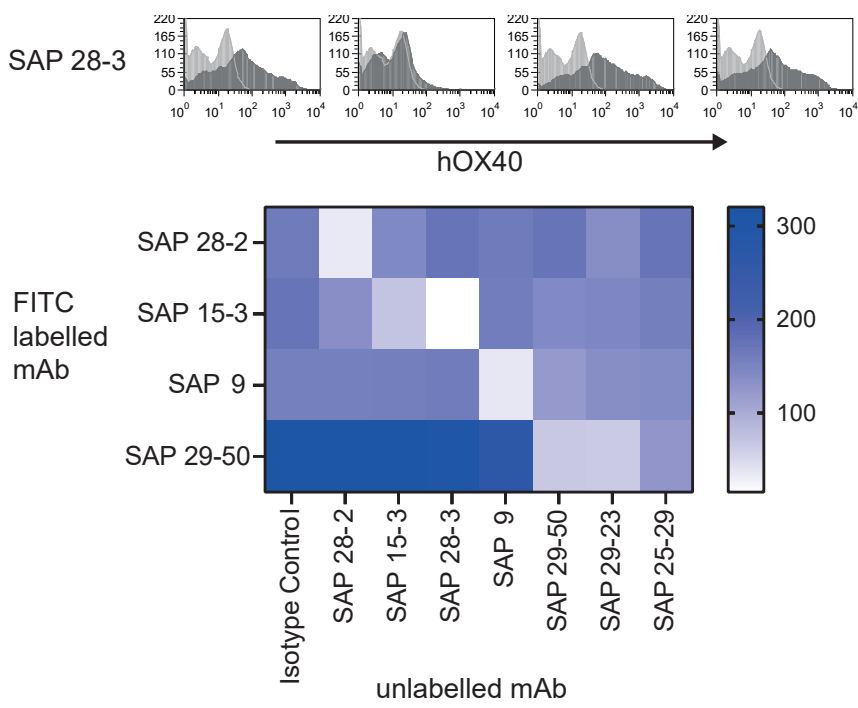

D.

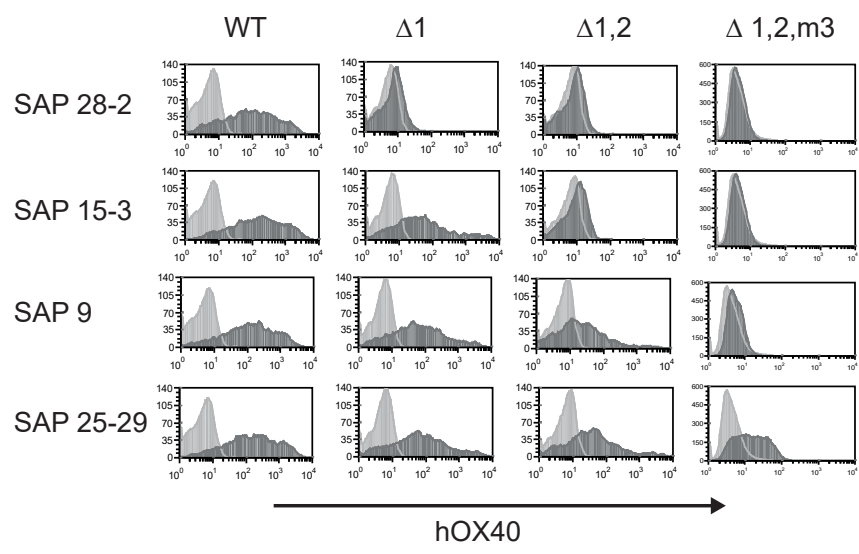

E.

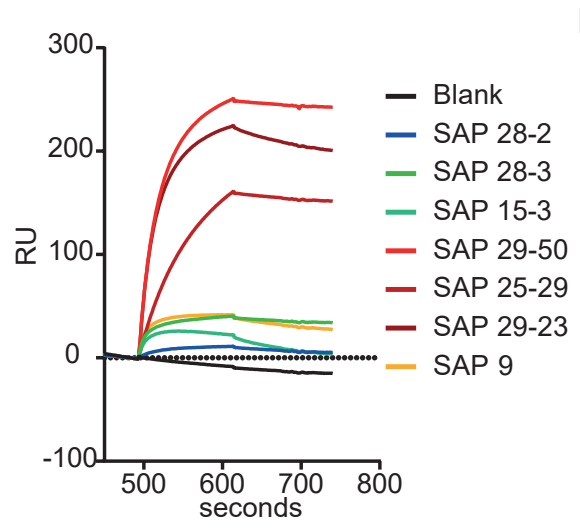

F.

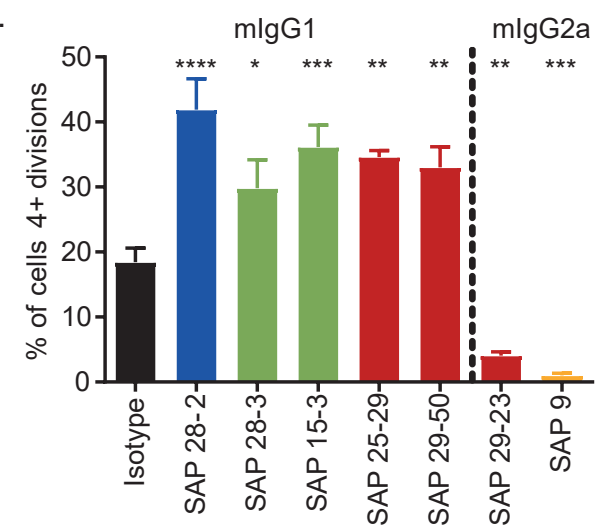


A. $\quad h O X 40 \mathrm{~K}^{+/-} \mathrm{OT}-\mathrm{I}$

hOX40KI+/ OT-I
splenocytes

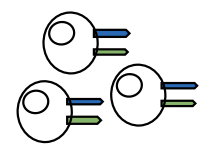

$\longrightarrow \mathrm{COX} 40$

mox40

B.

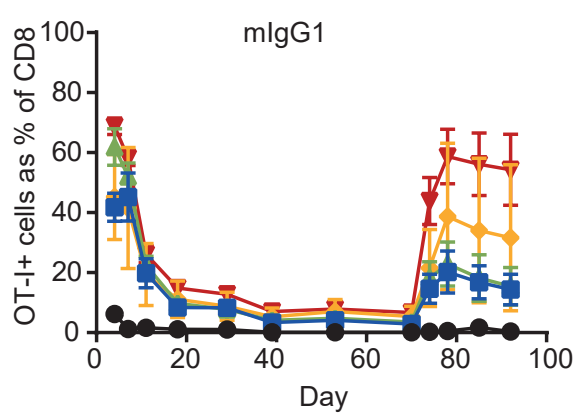

C.

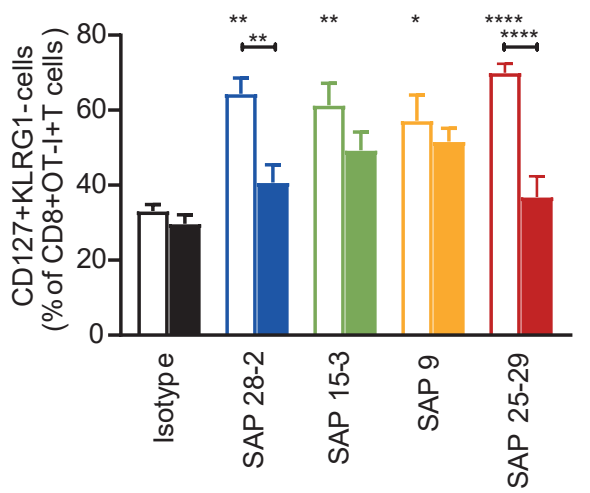

Kinetic analysis via blood sampling

Day 72

- SAP 28-2

- SAP 15-3

- SAP 9

* SAP 25-29

D.

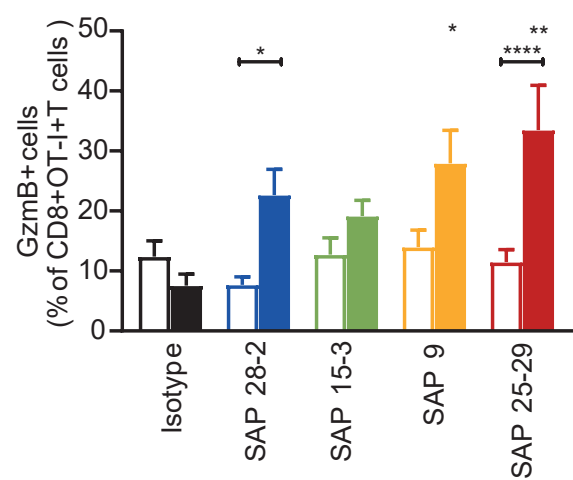

口 $\mathrm{mlgG1}$

- mlgG2a

mlgG1

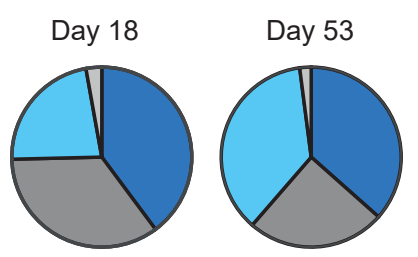

Day 74

Day 78

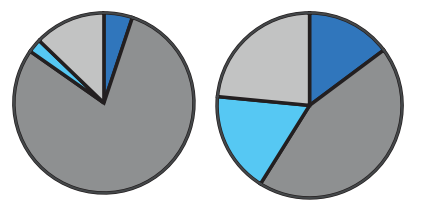

CXCR3 $3^{\mathrm{h}} \mathrm{CD} 43^{10}$

CXCR3 ${ }^{\text {hi }} C D 43^{\text {hi }}$

- $\mathrm{CXCR}^{\mathrm{lo}} \mathrm{CD} 43^{10}$ $\square \mathrm{CXCR}^{\mathrm{lo}} \mathrm{CD} 43^{\mathrm{hi}}$

mlgG2a
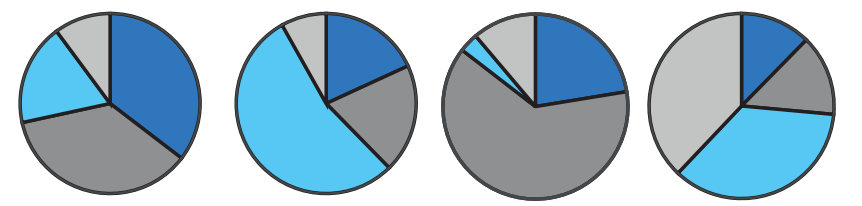

CXCR3 $3^{\text {hi }} \mathrm{CD} 43^{\text {lo }}$

口 CXCR3 ${ }^{\text {hi }} \mathrm{CD} 43^{\text {hi }}$

口 $\mathrm{CXCR}^{10} \mathrm{CD} 43^{10}$

$\square$ CXCR3 ${ }^{\text {lo }} \mathrm{CD} 43^{\text {hi }}$

E.
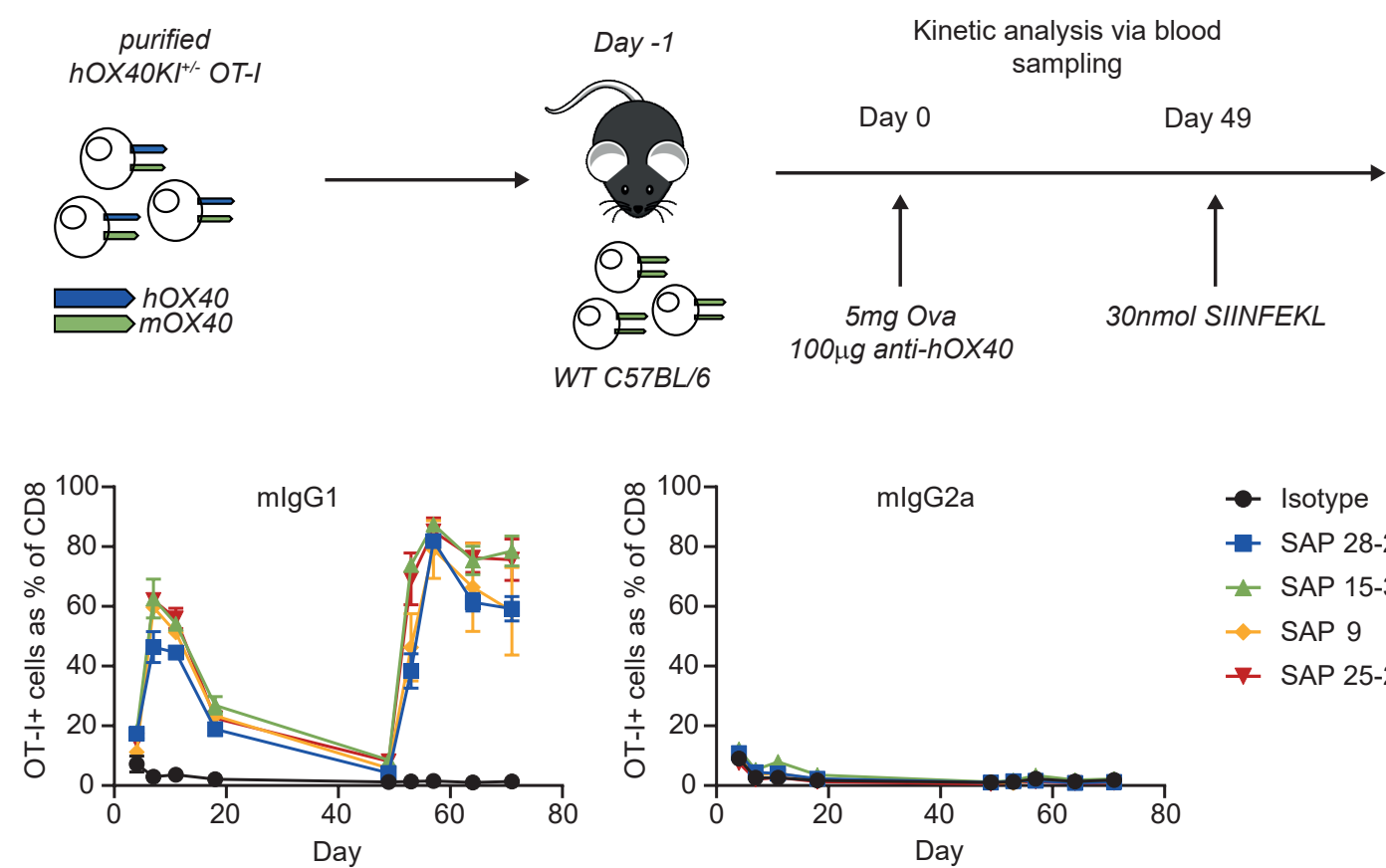

- Isotype

$\rightarrow$ SAP 28-2

\pm SAP 15-3

$\rightarrow$ SAP 9

$*$ SAP 25-29 
A

hOX40KI+/ OT-I

Day -1

splenocytes
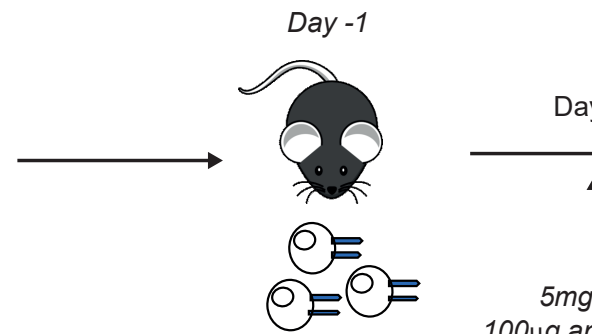

Day 0

Day 4

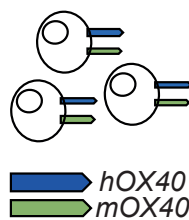

hOX40Kl ${ }^{+/+}$

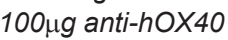

Spleens

B
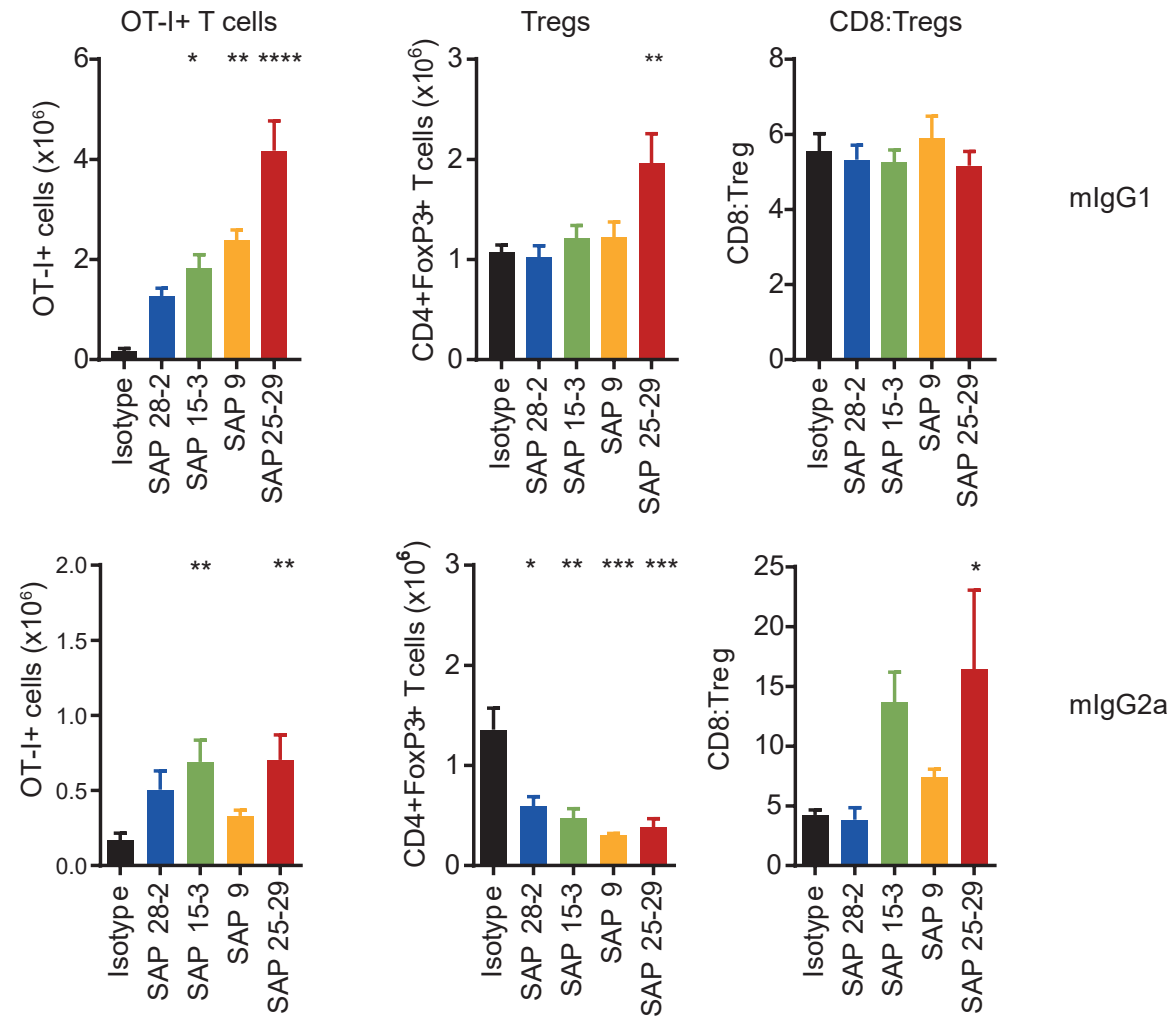

C

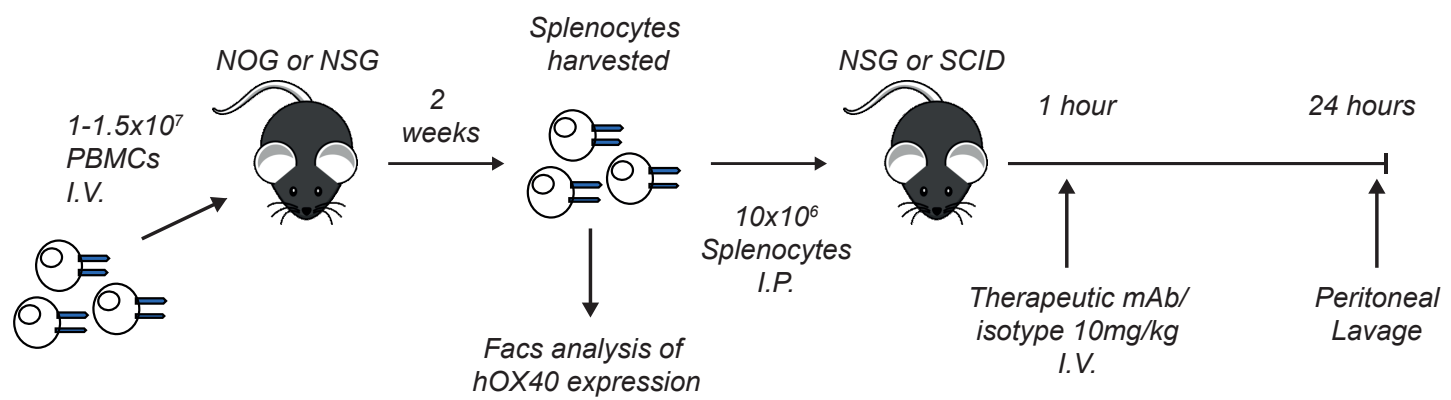

E
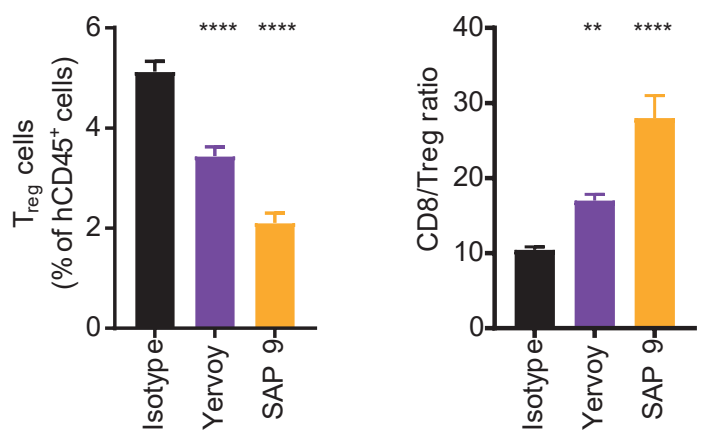

F

G
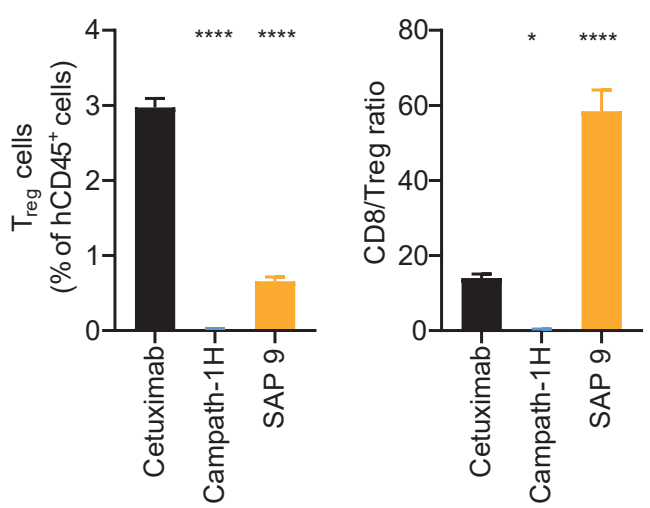
A
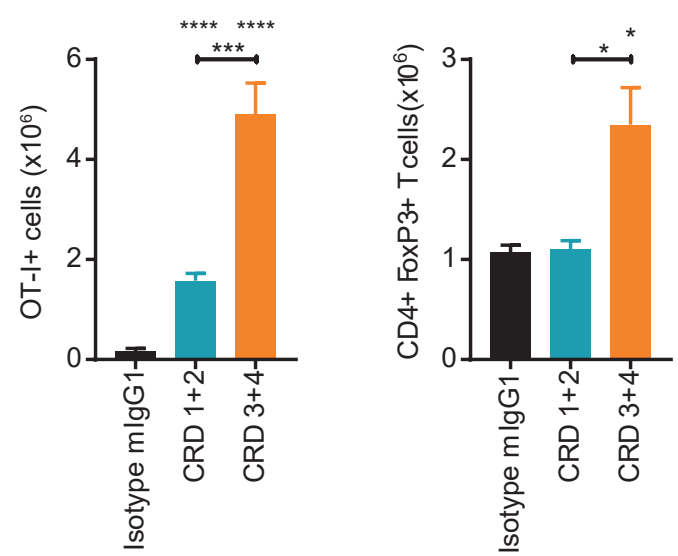

B
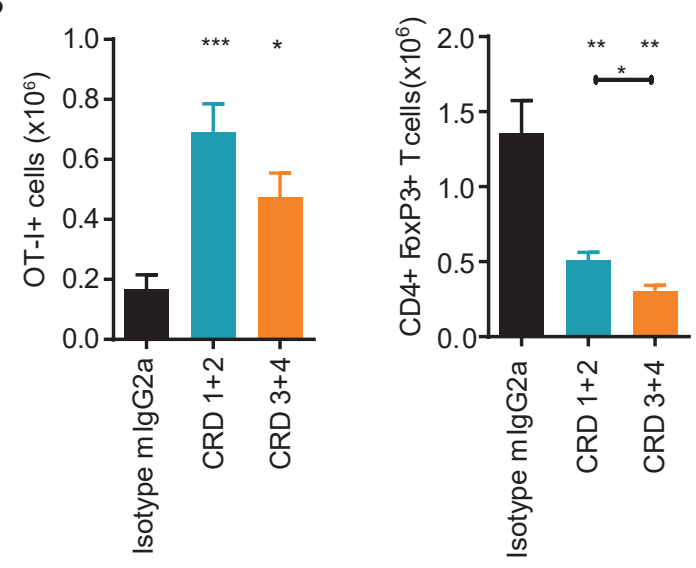

C
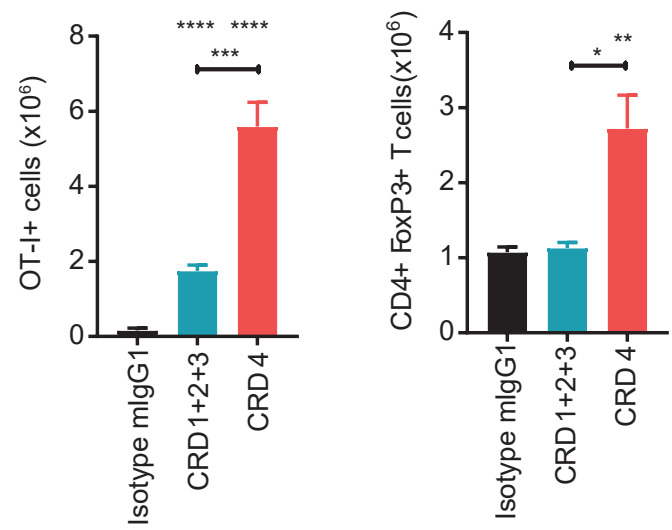

D
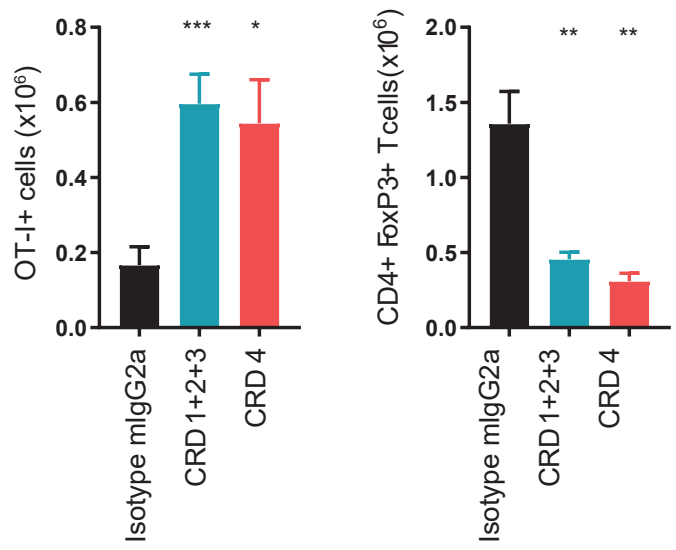
$A$

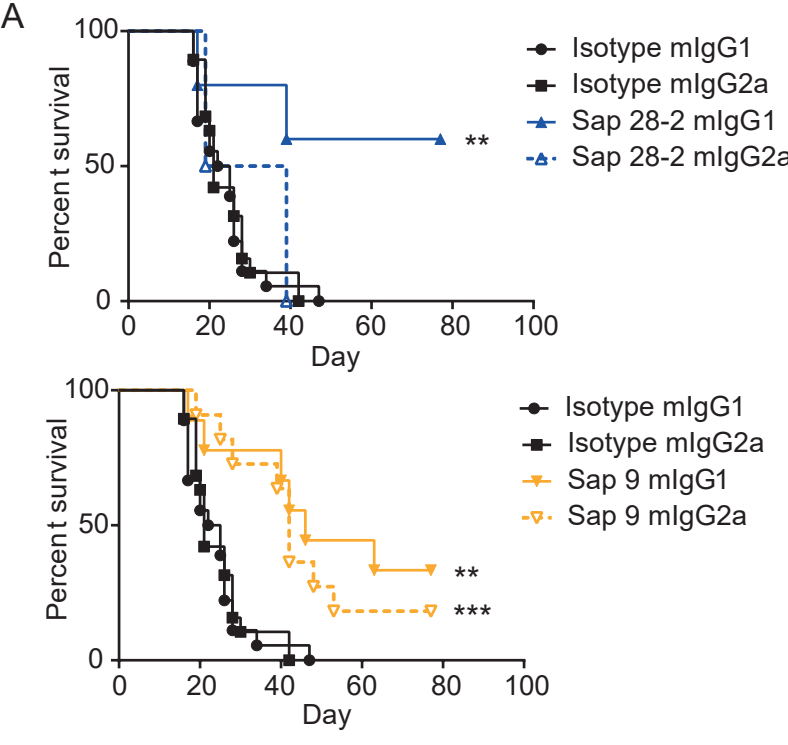

B
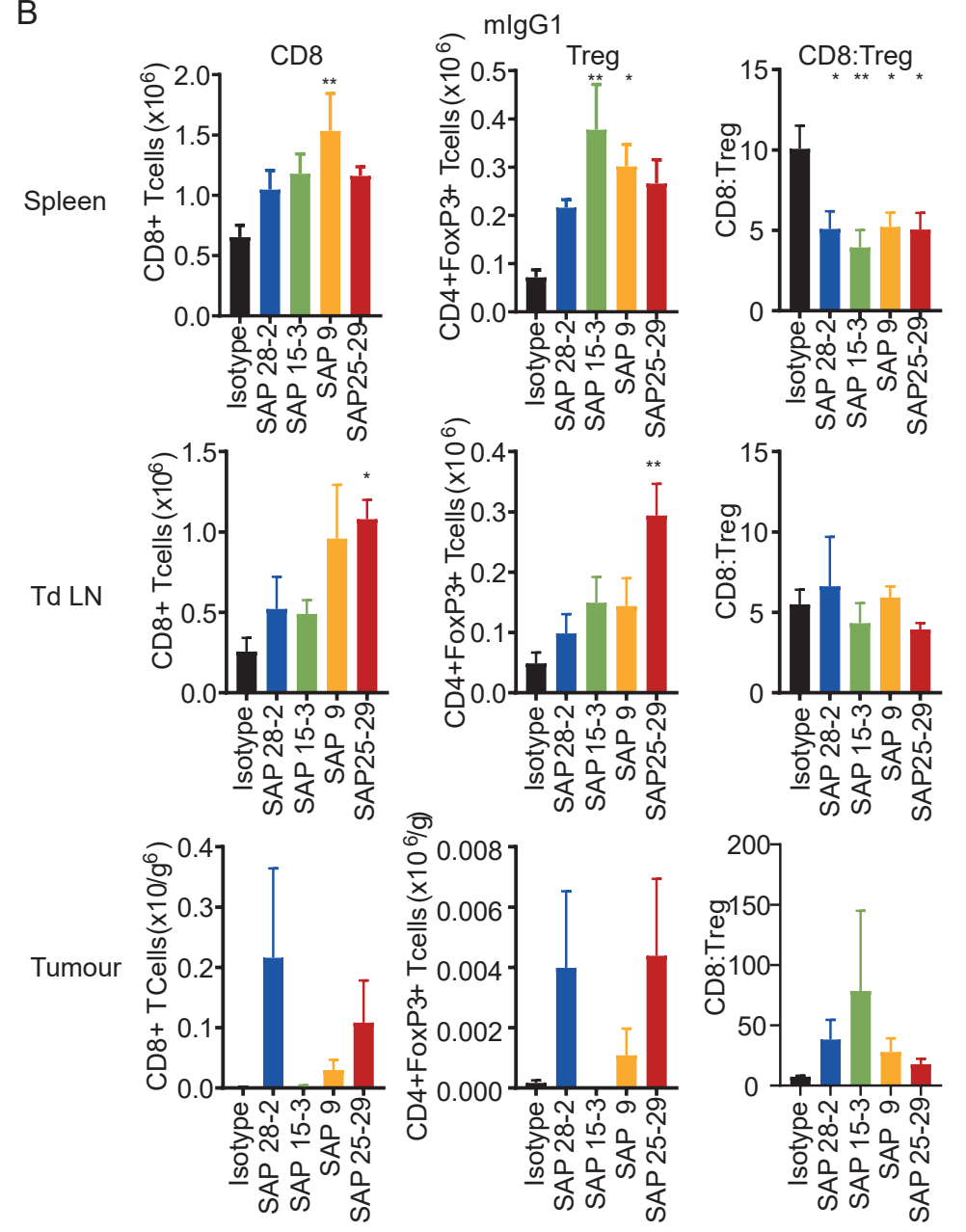

C
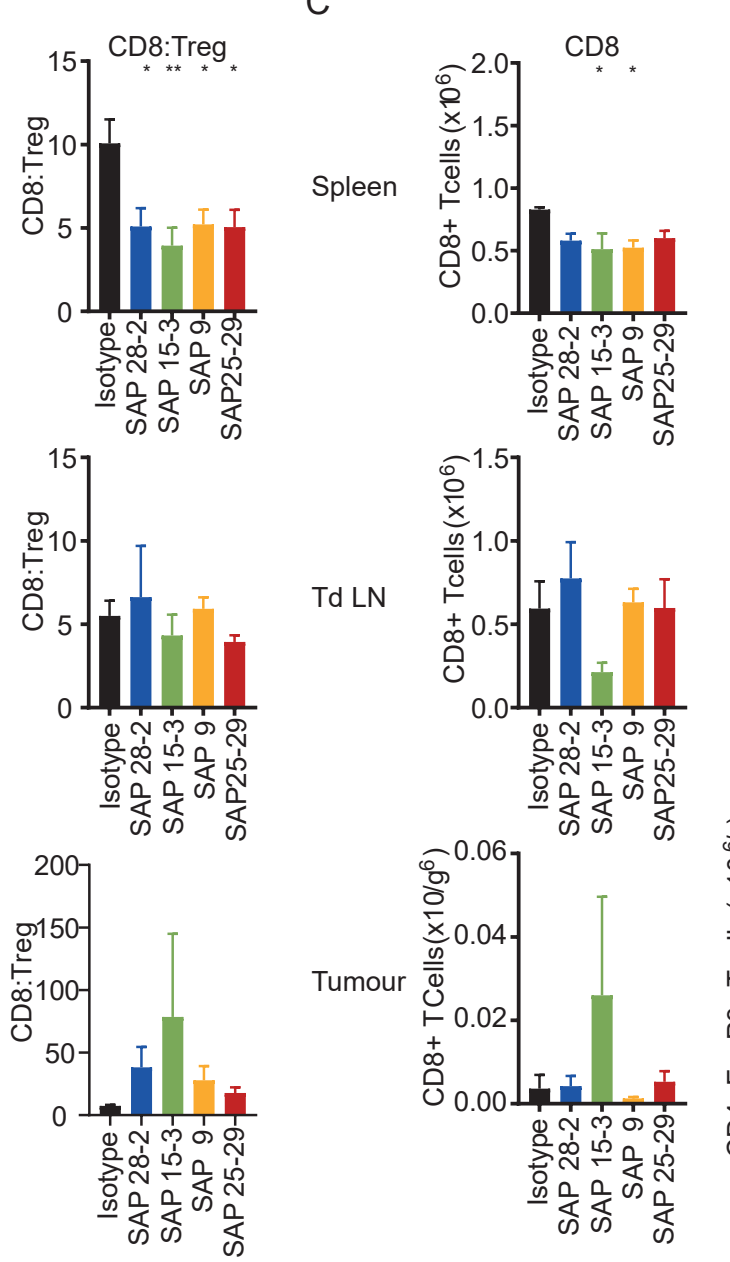

Td LN
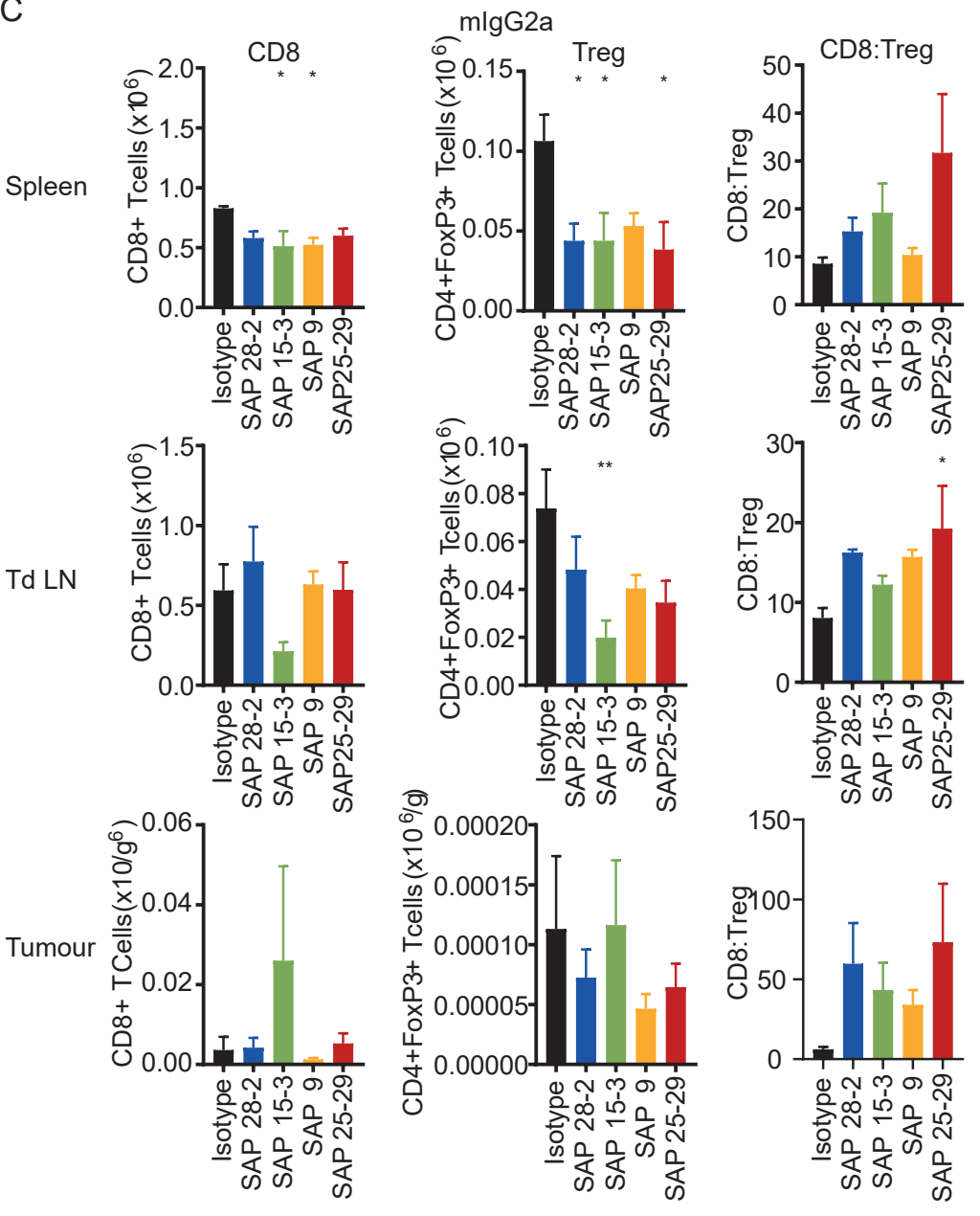

E
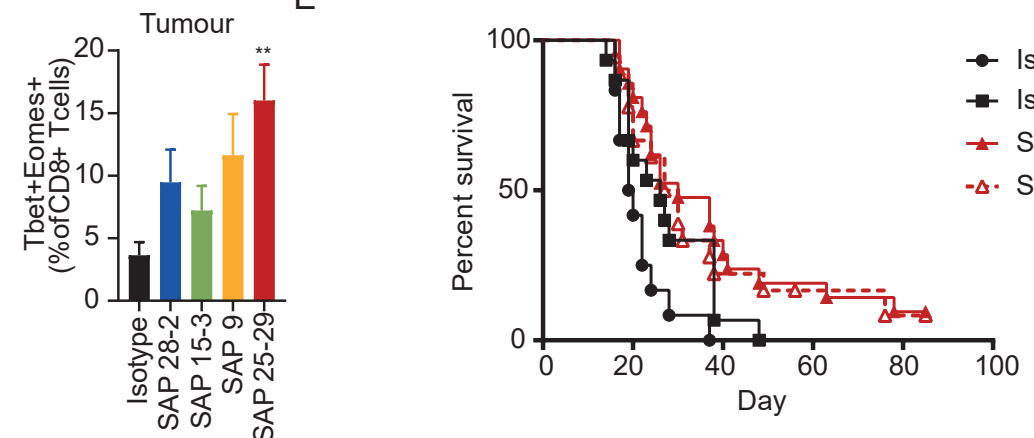

- Isotype mlgG1

sotype mlgG2a

.

mlgG1
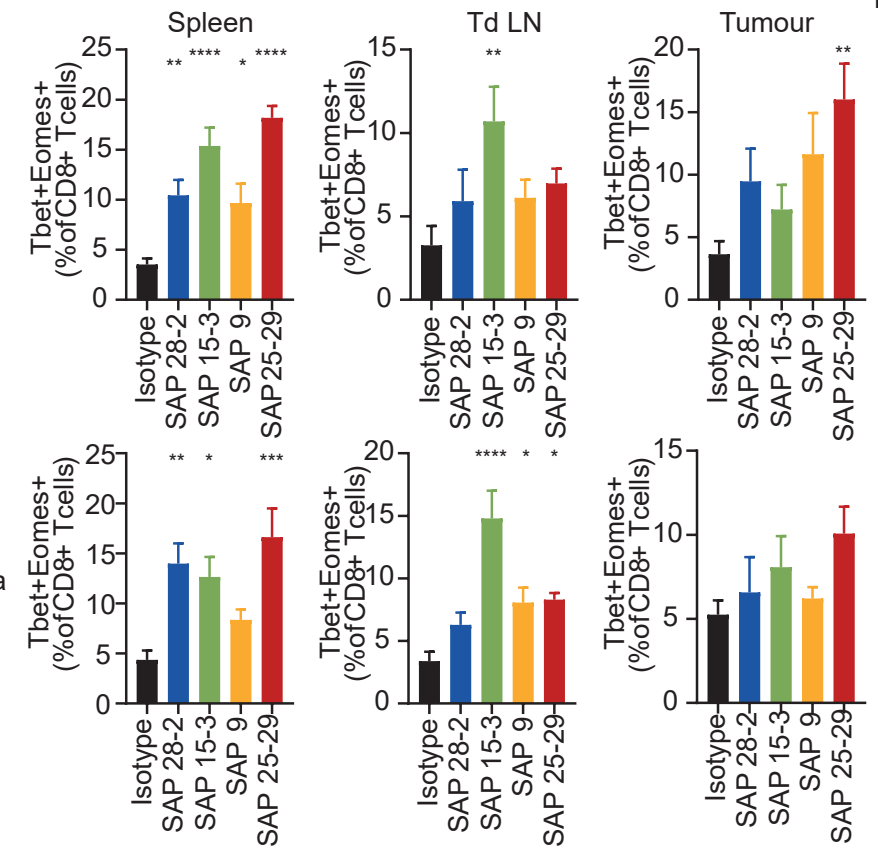

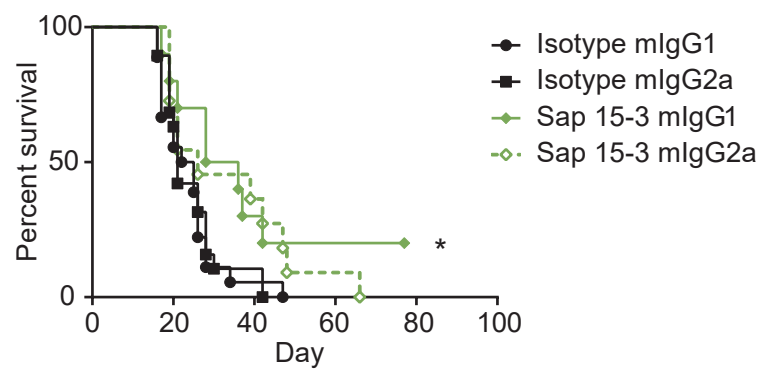

- Isotype mlgG2a

- Sap 25-29 mlgG1 -o. Sap 25-29 mlgG2a

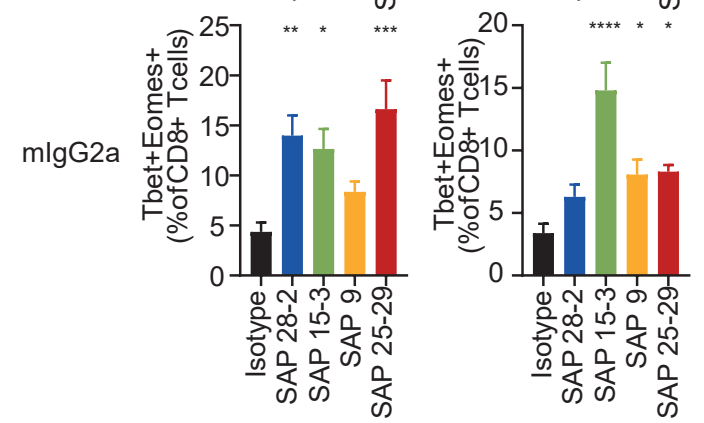




\begin{tabular}{|c|c|c|}
\hline Target & Clone & Company \\
\hline $\mathrm{mOX} 40$ & OX-86 & ThermoFisher-eBioscience \\
\hline $\mathrm{mCD} 8 \mathrm{a}$ & $53-6.7$ & ThermoFisher-eBioscience \\
\hline mCD4 & GK1.5 & ThermoFisher-eBioscience \\
\hline $\mathrm{mCD} 3$ & $145-2 C 11$ & ThermoFisher-eBioscience \\
\hline mNK1.1 & PK136 & ThermoFisher-eBioscience \\
\hline mFoxp3 & FJK-16s & ThermoFisher-eBioscience \\
\hline mB220 & RA3-6B2 & ThermoFisher-eBioscience \\
\hline $\mathrm{mCD} 11 \mathrm{c}$ & N418 & ThermoFisher-eBioscience \\
\hline $\mathrm{mF} 4 / 80$ & BM8 & ThermoFisher-eBioscience \\
\hline mCD11b & M1/70.15 & ThermoFisher-eBioscience \\
\hline $\mathrm{mCD} 62 \mathrm{~L}$ & MEL-14 & ThermoFisher-eBioscience \\
\hline $\mathrm{mCD} 44$ & IM7 & ThermoFisher-eBioscience \\
\hline mKLRG1 & $2 \mathrm{~F} 1$ & ThermoFisher-eBioscience \\
\hline mCD127 & A7R34 & ThermoFisher-eBioscience \\
\hline mCXCR3 & CXCR3-173 & ThermoFisher-eBioscience \\
\hline mCD45.2 & 104 & ThermoFisher-eBioscience \\
\hline mT-bet & eBio4B10 & ThermoFisher-eBioscience \\
\hline mEOMES & DAN11MAG & ThermoFisher-eBioscience \\
\hline mCD43 (activation glycoform) & 1B11 & BioLegend \\
\hline mLy6C & HK1.4 & BioLegend \\
\hline mLy6G & 1A8 & BioLegend \\
\hline h/m Granzyme B & GB11 & ThermoFisher-Invitrogen \\
\hline $\mathrm{H}-2 \mathrm{~K}^{\mathrm{b}} / \mathrm{SIINFEKL}$ tetramer & & In house \\
\hline hCD4 (healthy samples) & RPA-T4 & ThermoFisher-eBioscience \\
\hline hCD8 (healthy samples) & SK1 & ThermoFisher-eBioscience \\
\hline hOX40 (healthy samples) & ACT35 & ThermoFisher-eBioscience \\
\hline hCD127 (healthy samples) & eBioRDR5 & ThermoFisher-eBioscience \\
\hline hFoxp3 (healthy samples) & 236A/E7 & ThermoFisher-eBioscience \\
\hline hCD56 (healthy samples) & CMSSB & ThermoFisher-eBioscience \\
\hline hCD14 (healthy samples) & 61D3 & ThermoFisher-eBioscience \\
\hline hCD3 (healthy samples) & SK7 & BioLegend \\
\hline hCD25 (healthy samples) & M-A251 & BioLegend \\
\hline hCD19 (healthy samples) & HIB19 & BioLegend \\
\hline $\begin{array}{l}\text { hCD4 (ovarian cancer and } \\
\text { human cells in NSG samples) }\end{array}$ & RPA-T4 & BD Biosciences \\
\hline $\begin{array}{l}\text { hCD25 (ovarian cancer and } \\
\text { human cells in NSG samples) }\end{array}$ & M-A251 & BD Biosciences \\
\hline $\begin{array}{l}\text { hCD127 (ovarian cancer and } \\
\text { human cells in NSG samples) }\end{array}$ & HIL-7R-M21 & BD Biosciences \\
\hline $\begin{array}{l}\text { hCD8 (ovarian cancer and } \\
\text { human cells in NSG samples) }\end{array}$ & RPA-T8 & BD Biosciences \\
\hline $\begin{array}{l}\text { hOX40 (ovarian cancer and } \\
\text { human cells in NSG samples) }\end{array}$ & ACT35 & BD Biosciences \\
\hline isotypes & & Corresponding companies \\
\hline $\begin{array}{l}\text { fixable eFluor780 Live/Dead } \\
\text { stain }\end{array}$ & & ThermoFisher-eBioscience \\
\hline aqua live/dead viability stain & & ThermoFisher-Invitrogen \\
\hline
\end{tabular}


1 Supplementary Methods

3 PCR genotyping of mice

4 hOX40 $\mathrm{KI}$ was detected using the following primers $\mathrm{F}$ -

5 AGTGCCCACGCTTCCTGAGGA and R - CTTGAGGATGCCAGAGGAGGC

6 which give a 290bp product. mOX40 was detected using the following primers

7 F -TCTCCACCCACCTTGGTGACT and R - GCCAGCAGGACAGTCAAGGA

8 which give a $174 \mathrm{bp}$ product.

Antibody Production and labelling

11 Anti-hOX40 mAb were created using conventional hybridoma technology [49].

12 Isotype switching was carried out as previously described [50] and mAb

13 purified either using a protein-G or a protein-A column. All preparations were

14 deemed endotoxin low (<1ng/mg protein). Anti-hOX40 mAb were FITC

15 (Sigma) labelled at a ratio of 1:10 FITC:mAb and purified using a desalting column (GE Healthcare).

Surface Plasmon Resonance

19 A Biacore T100 upgraded to a T200 (GE Life Sciences) was used to measure 20 interactions with hOX40. For affinity determination $1 \mu \mathrm{g} / \mathrm{ml}$ of hOX40-hFc was 21 immobilised onto a CM5 chip (GE Healthcare) and a range of concentrations 22 (0-500nM) of anti-hOX40 mAb was injected over the chip. For ligand binding 23 to hOX 40; $1 \mu \mathrm{g} / \mathrm{ml}$ of hOX40-hFc was immobilised onto a CM5 chip coated 24 with an anti-Fc antibody and hOX40L, mOX40L, h4-1BBL or m4-1BBL $25(15 \mu \mathrm{g} / \mathrm{ml})$ was injected over the chip.

In vitro assays hPBMC proliferation assays: hPBMCs were isolated using lymphoprep separation. For proliferation assays cells were labelled with $1 \mu \mathrm{M}$ CFSE

30 immediately following isolation, cultured at high density $\left(1.5 \times 10^{7}\right.$ cells $\left./ \mathrm{ml}\right)$ for 2

31 days at $37^{\circ} \mathrm{C}$, then plated at $1 \times 10^{5}$ cells/well. Cells were stimulated with

32 soluble anti-CD3 (OKT3, $5 \mathrm{ng} / \mathrm{ml})$ and anti-hOX40 mAb $(5 \mu \mathrm{g} / \mathrm{ml})$. Cells were 
33 stained with appropriate antibodies and CFSE dilution assessed via flow

34 cytometry. Murine expression assays; splenocytes were isolated using a $100 \mu \mathrm{m}$ strainer and red cell lysed using ACK lysis buffer. Cells were plated at $2 \times 10^{5}$ cells/well and stimulated with soluble anti-CD3 (145-2C11, 0.1 $\mathrm{g} / \mathrm{ml})$ and anti-CD28 $(37.51,1 \mu \mathrm{g} / \mathrm{ml})$. Cells were stained with appropriate antibodies and analysed by flow cytometry.

Murine proliferation assays; splenocytes were isolated using a $100 \mu \mathrm{m}$ strainer and red cell lysed using ACK lysis buffer. Cells were labelled with $5 \mu \mathrm{M}$ CFSE, plated at $1 \times 10^{5}$ cells/well and stimulated with platebound anti-CD3 (145-2C11, $1 \mu \mathrm{g} / \mathrm{ml})$ and anti-OX40 mAb $(10 \mu \mathrm{g} / \mathrm{ml})$. Cells were incubated for 72 hours, stained with appropriate antibodies and analysed for CFSE dilution by flow cytometry.

\section{Flow cytometry}

Flow cytometry antibodies are listed in Supplementary Table 1. Intracellular staining was performed using Foxp3 staining buffer kit (ThermoFishereBioscience) according to the manufacturer's protocol. Cells from ovarian cancer patients were incubated with $10 \mathrm{mg} / \mathrm{ml} \mathrm{KIOVIG} \mathrm{(Baxalta)} \mathrm{prior} \mathrm{to}$ staining with relevant antibodies. All flow cytometry experiments were performed using either a FACSCalibur, FACSCanto, FACSAria or FACSVerse machine (all BD Bioscience). Data analysed using Cellquest Pro, FACSDiva (V6.1.2), FlowJo (all BD Bioscience) or FCS Express (V.3) software (De Novo Software). 
A

Targeted Allele
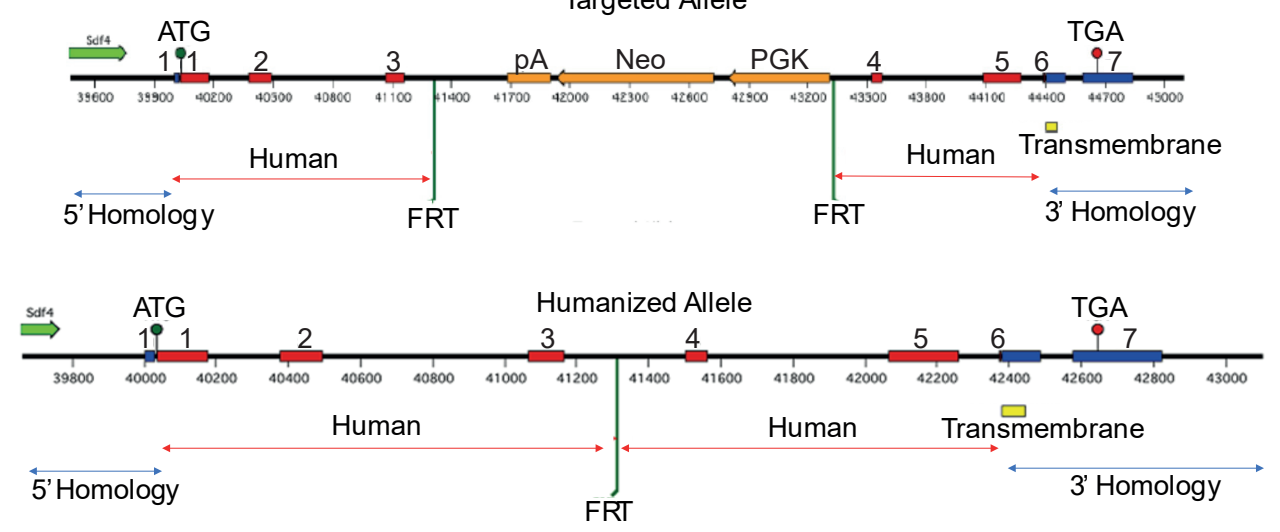

B

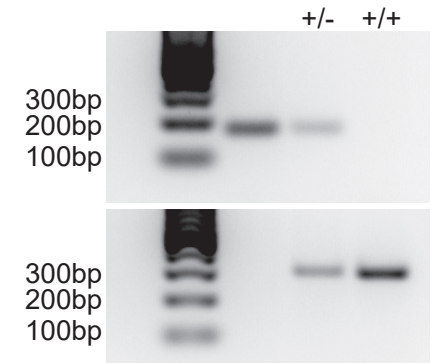

hOX4OKI PCR
C
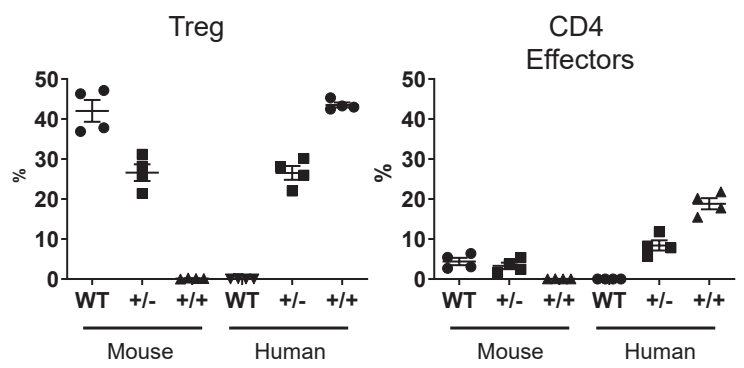

CD4

Human

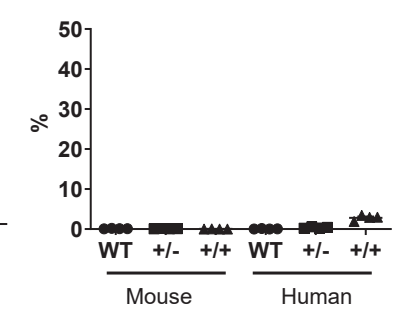

D

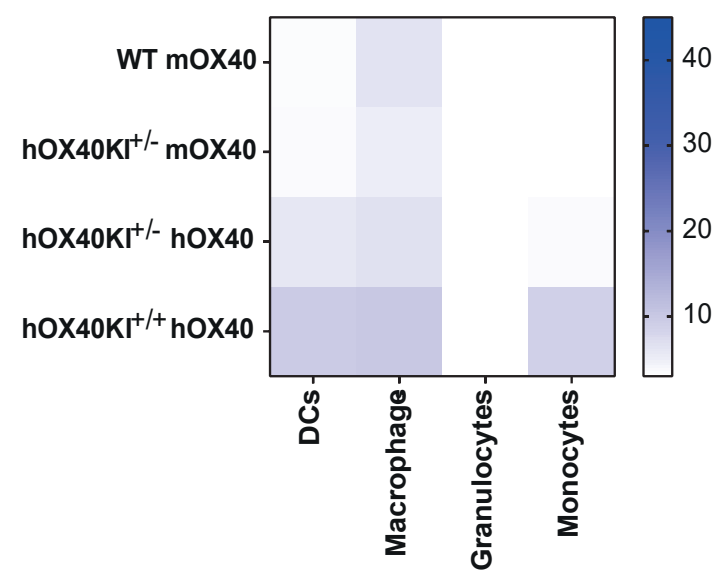

$E$
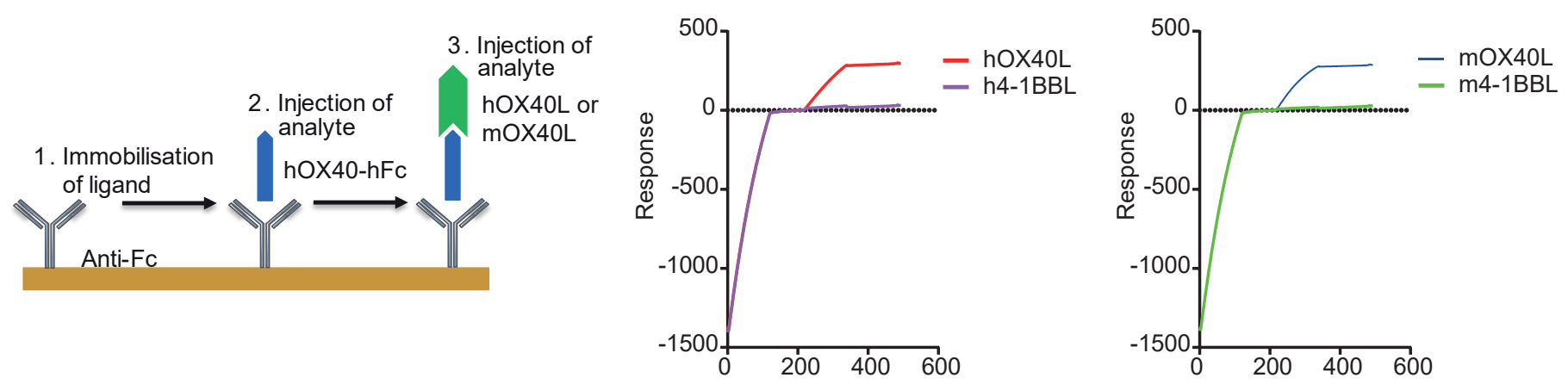

$\mathrm{F}$
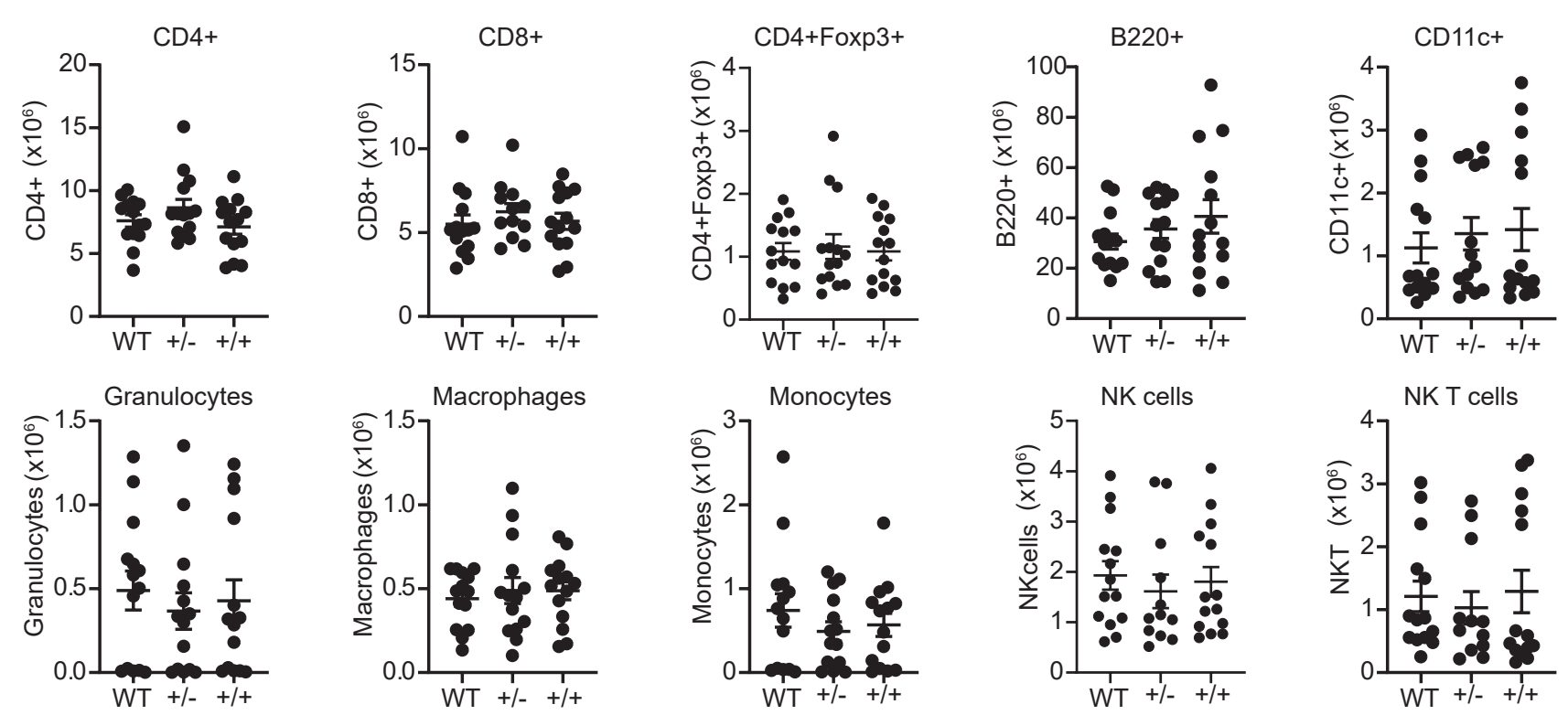

Supplementary Figure 1. hOX40 expression in hOX40KI mice is dose dependent. A. Schematic of hOX40 chimeric receptor construct used to generate the hOX40KI mice. B. PCR showing the genotyping of WT, hOX40KI+/- and hOX40KI ${ }^{+/+}$mice. C. Expression of mouse and human OX40 on Treg (left panel), CD4 effectors (middle panel) and CD8 T cells (right panel) isolated from WT, hOX40Kl+and $h O X 40 \mathrm{Kl}^{+/+} n=4$. D. Heat map showing expression levels on myeloid populations. E. SPR analysis of OX40 $L$ binding to $h O X 40$. Left panel shows schematic, h4-1BBL and m4-1BBL were used as negative controls. Middle panel shows hOX40L (red) and h4-1BBL (purple) binding and right panel shows mOX40L (blue) and m4-1BBL (green) F. Lymphocyte and Myeloid populations in WT, hOX$40 \mathrm{Kl}^{+/-}$and $\mathrm{hOX} 40 \mathrm{Kl}^{+/+}$mice $(6-10$ weeks age, $\mathrm{n}=14)$. 
mlgG1

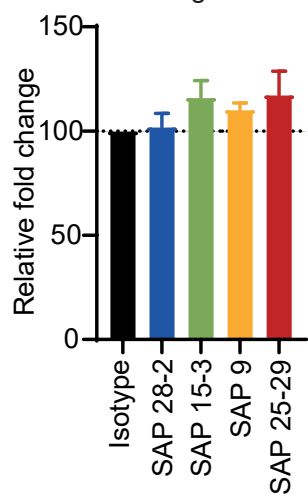

CD4

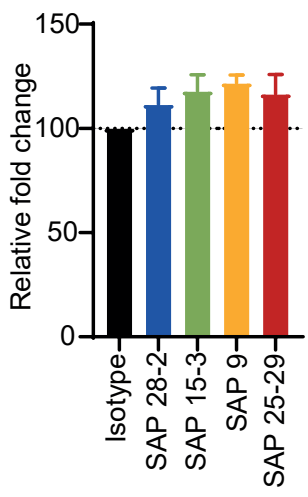

mlgG2a
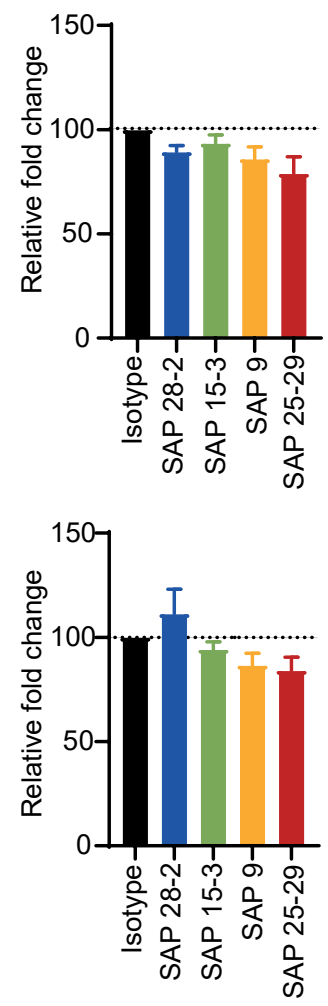

Supplementary Figure 3. anti-hOX40mAb mlgG1 promotes proliferation whilst mlgG2a reduces proliferation. hOX40KI ${ }^{+/+}$splenocytes were activated with $\alpha$ CD3 $(1 \mu \mathrm{g} / \mathrm{ml})$ and $\alpha$ hOX $40 \mathrm{mAb}(10 \mu \mathrm{g} / \mathrm{ml})$ for 72 hours and then CFSE dilution analysed. Data presented as fold change over isotype contol, $n=5$. 
A

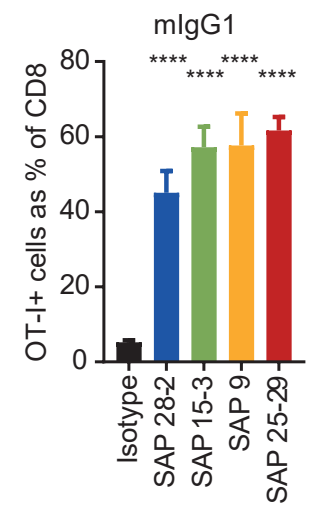

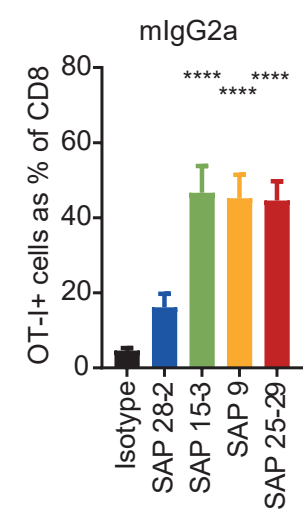

SAP 15-3
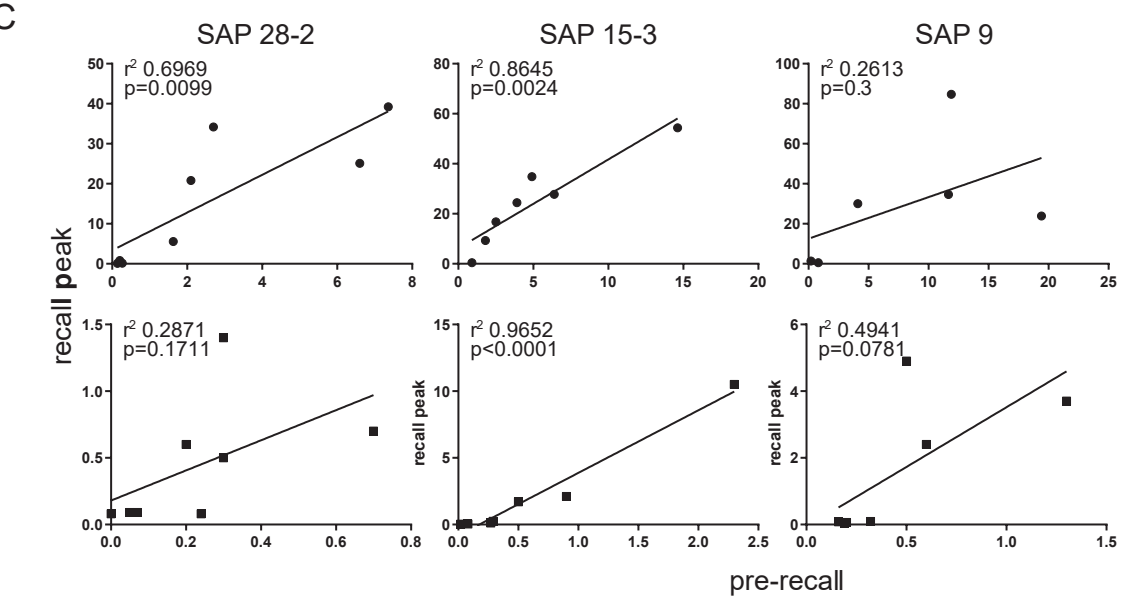

B
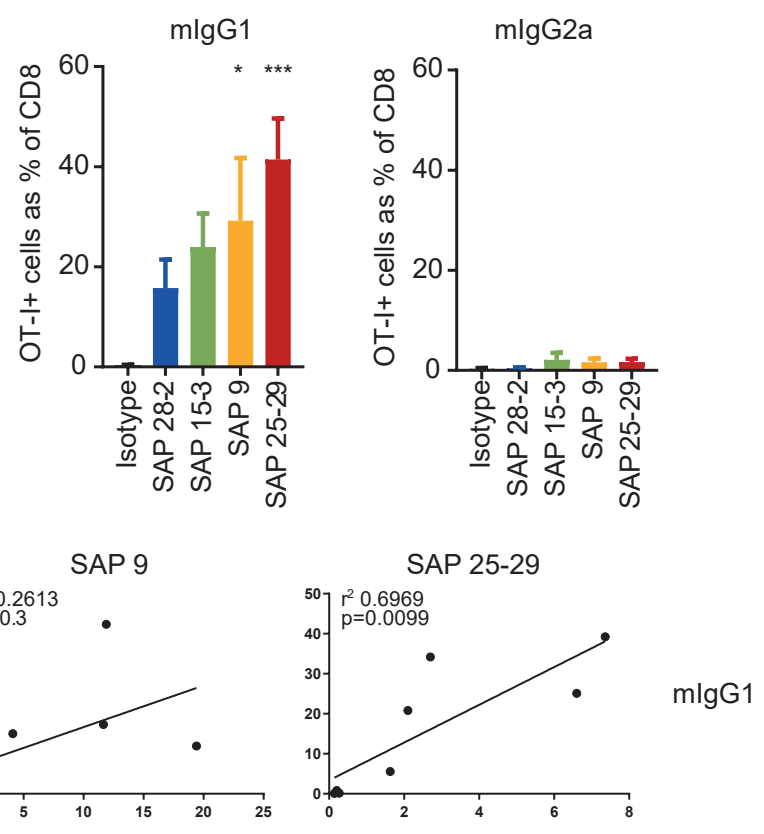

$\mathrm{D}$
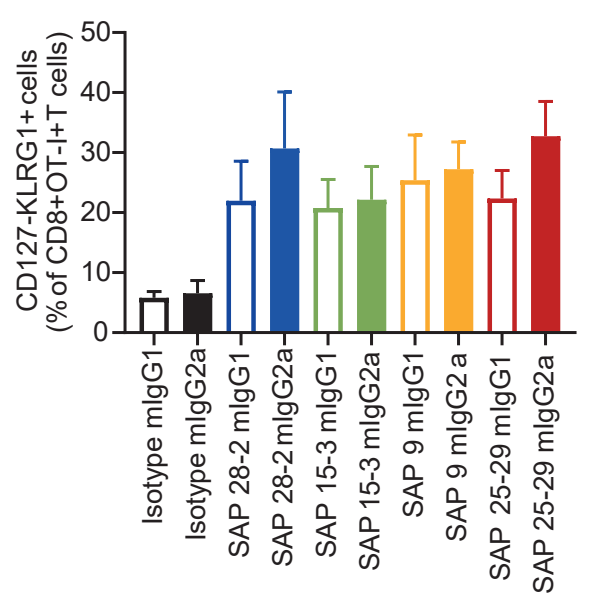

$\mathrm{E}$
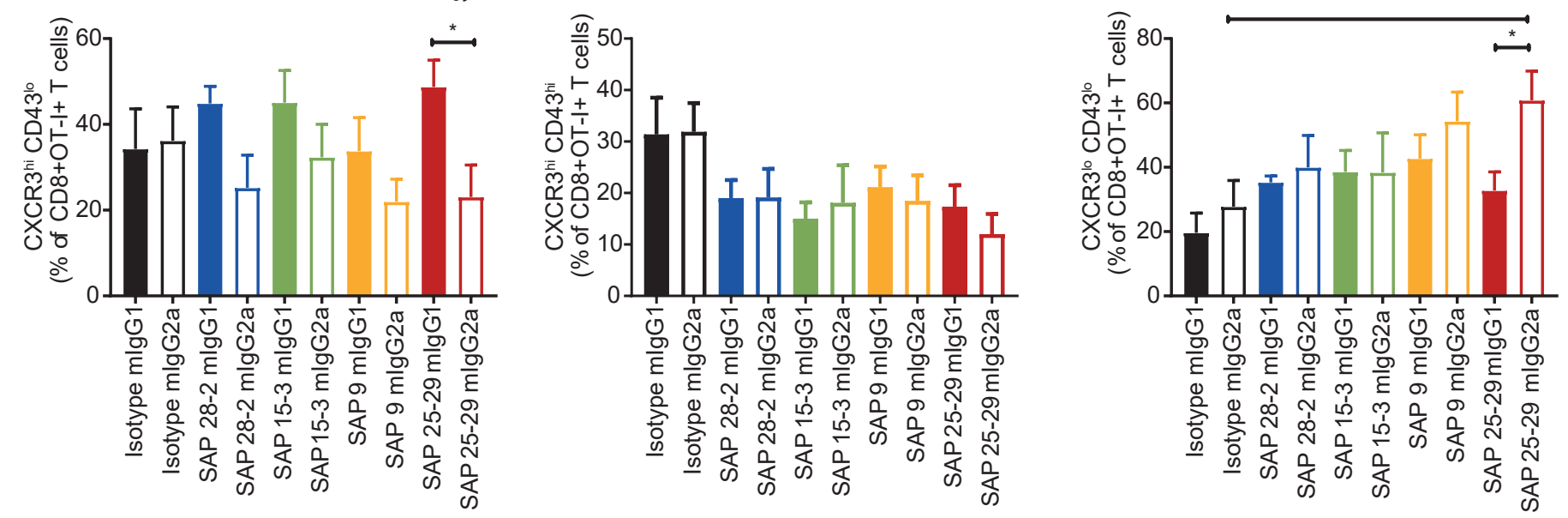

Supplemental Figure 4. anti-hOX40 mlgG1 act agonistically in vivo. A. Primary peak response of OT-I expansion in blood to anti-hOX40 mlgG1 (left panel) or mlgG2a (right panel) ( $n=6-8$, pooled from 2 independent experiments). B. Recall peak following SIINFEKL rechallenge of mice previously treated with anti-hOX40 mlgG1 (left panel) or mlgG2a (right panel) ( $\mathrm{n}=6-8$, pooled from two independent experiments). C. Correlation graphs between recall response and pre-recall OT-I levels in anti-hOX40 mlgG1 (top row) and $\mathrm{mlgG} 2 \mathrm{a}$ (bottom row) treated mice. $\mathrm{n}=6-8$, pooled from two independent experiments. D. Analysis of OT-I SLECs CD127-KLRG1+ in the blood at D18 $(n=8)$. E. CXCR3 and CD43 analysis of OT-I in the blood pre-rechallenge with SIINFEKL peptide ( $\mathrm{n}=7-8$ pooled from 2 independent experiments). CXCR3hiCD43lo (left panel), CXCR3hiCD43hi (middle panel) and CXCR3loCD43lo (right panel). ${ }^{*} p<0.05$ Sidak's test. 
A

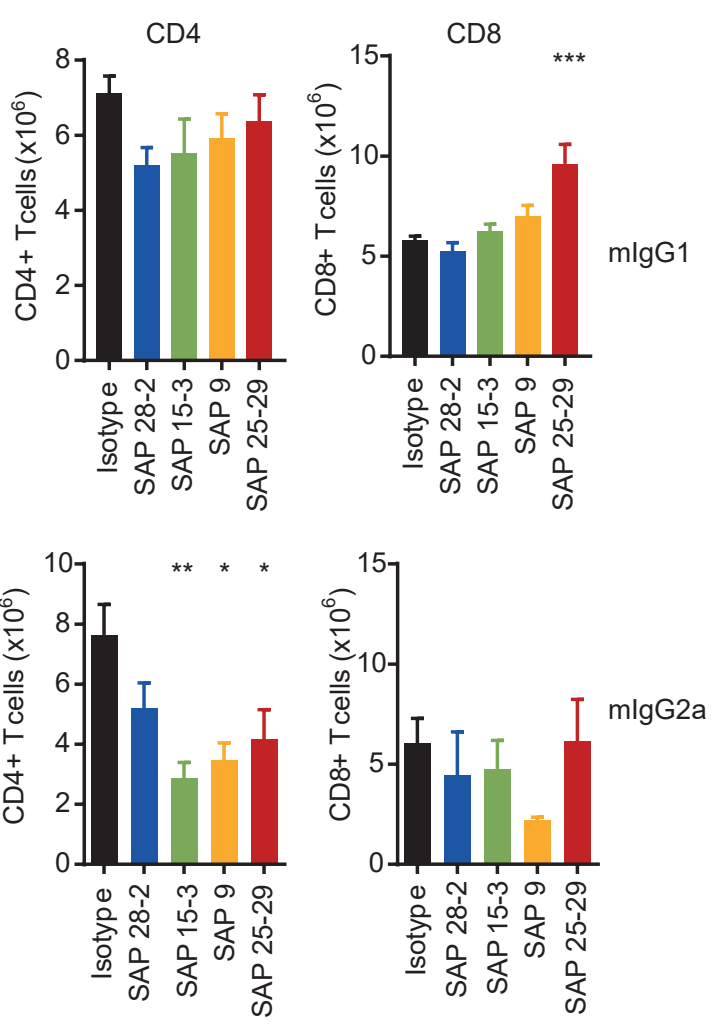

B

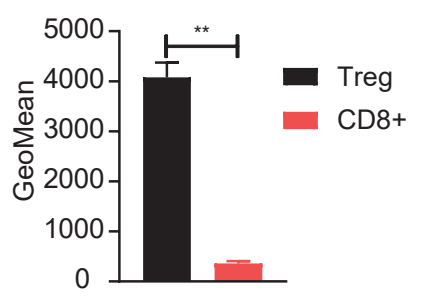

Supplemental Figure 5. Isotype of anti-hOX40 mAb influences effector function. A. CD4 and CD8 T cell numbers assessed on Day 4 in splenocytes isolated from hOX $40 \mathrm{Kl}^{+/+}$mice receiving $1 \times 10^{5} \mathrm{hOX} 40 \mathrm{KI}^{++} \mathrm{OT}-\mathrm{I}$ followed by $100 \mu \mathrm{g}$ anti-hOX40 mAb as either a mlgG1 or mlgG2a ( $n=7-8$, pooled from 2 independent experiments). ${ }^{* *} p<0.01$, * $p<0.05$ Dunnett's test. B. hOX40 expression on Treg and CD8+ T cells isolated from NOG mice 2 weeks post injection of hPBMCs ( $n=5$, representative of 2 independent experiments). ${ }^{* *} p<0.01$ Mann Whitney $U$ test. 


\section{A}

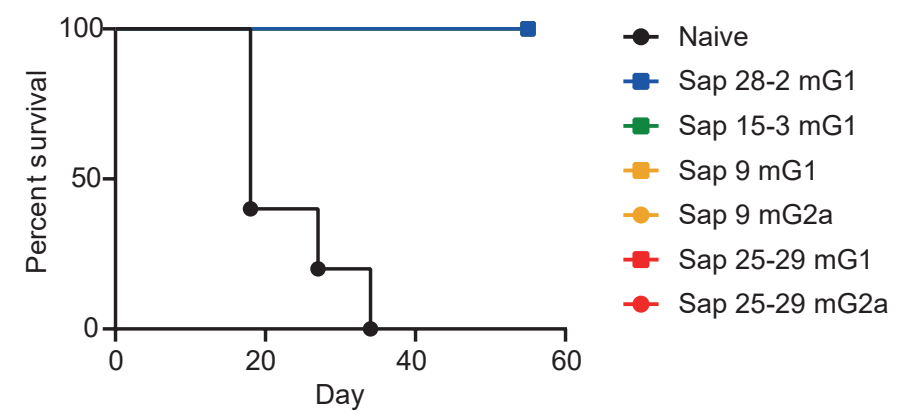

B

mlgG1
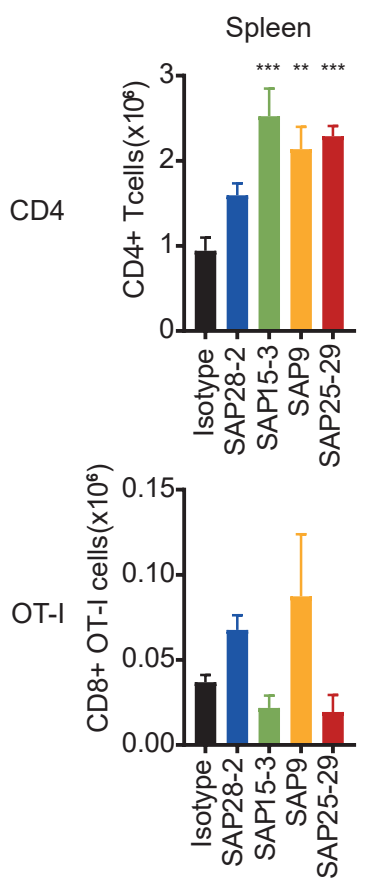

Tumour
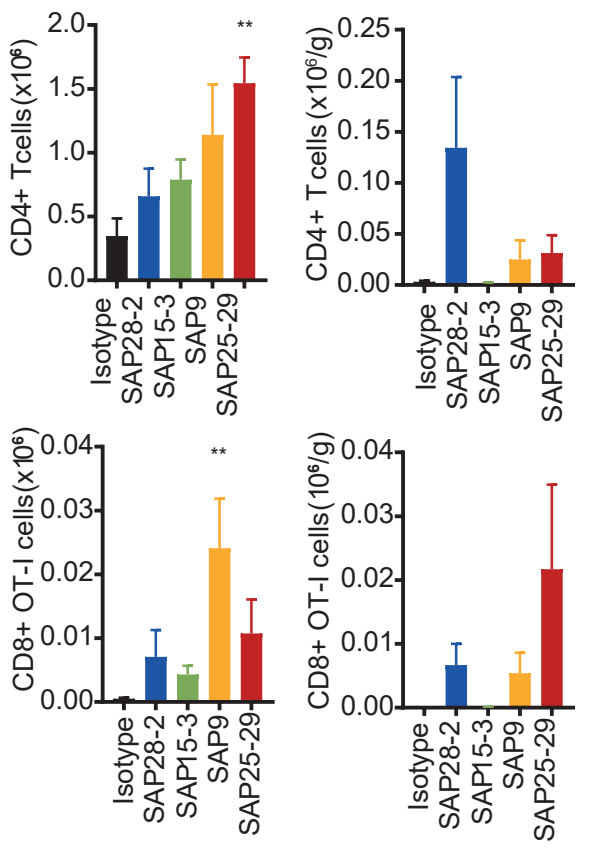

C

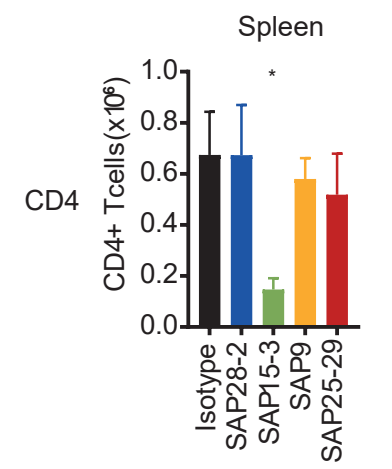

mlgG2a

Td LN
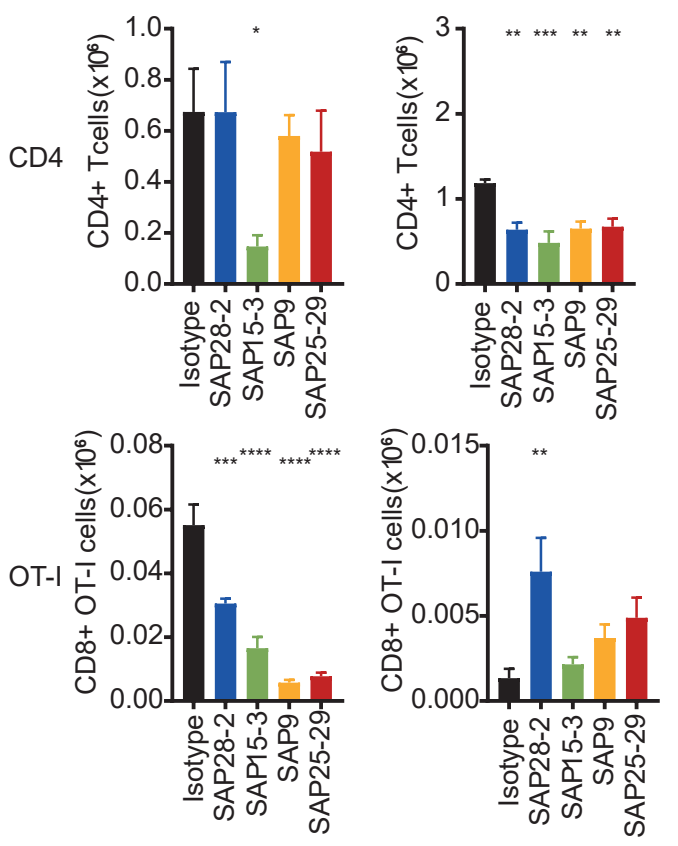

Tumour
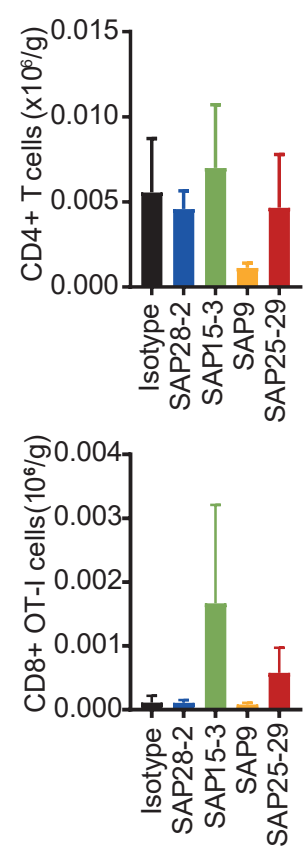

Supplementary Figure 6. hOX40mAb are therapeutic as both mlgG1 and mlgG2a. A. Survival graphs of mice rechallenged with E.G7 lymphoma cells shown alongside naive recipients. $n=1-5$ representative of 2 independent experiments. $B$ and $C$. Assessment of CD4+ (top rows) and CD8+OT-I (bottom rows) T cell populations in Spleen (left panels), Tumour draining lymph node (middle panels), and Tumour (right panels) isolated 24 hours post second mAb dose either as a mlgG1 (B) or mlgG2a (C). $n=3-5$, representative of 2 independent experiments. ${ }^{* * *} p<0.0001,{ }^{* * *} p<0.001{ }^{* *} p<0.01{ }^{*} p<0.05$ Log-rank (Mantel-Cox) for survival graphs A and Dunnett's multiple comparison test for B-C. 
